Classification

Physics Abstracts

$07.50-89.29-83.10 \mathrm{D}$

\title{
Modélisation dynamique du moteur pas à pas
}

\author{
Michel Kant et Jean-Paul Vilain \\ Laboratoire d'Electromécanique, Université de Technologie de Compiègne, France
}

(Reçu le 18 mai 1989, révisé le 11 octobre 1989, accepté le 30 mars 1990)

\begin{abstract}
Résumé. - Le mémoire se propose de systématiser la modélisation dynamique de tous les moteurs pas à pas, de manière à lever un certain nombre d'ambiguïtés sur leur technologie et surtout sur leur pilotage. La modélisation passe par trois étapes essentielles : équations dans les coordonnées immobiles, équations dans les coordonnées mobiles et finalement équations adimensionnelles. Dans cette dernière écriture, tous les actionneurs pas à pas présentent réellement une structure analytique unique. Les équations du mouvement étant non linéaires, leur résolution ne peut être que numérique. La programmation correspondante fait apparaître clairement les propriétés physiques des différentes étapes de fonctionnement: comme le positionnement sur un pas, le mouvement quasidynamique et le mouvement dynamique. On montre, par ailleurs, l'influence des paramètres de construction sur l'espace de fonctionnement de la machine. Un certain nombre de ces considérations ont déjà été présentées, notamment dans [1, 5-8], par des modélisations physiques partielles, relatives aux structures et aux alimentations particulières. Notre démarche, outre la globalisation des résultats, doit contribuer à unifier la théorie des machines pas à pas, d'une part, et indiquer le repère approprié pour les études spécifiques, d'autre part.
\end{abstract}

\begin{abstract}
The aim of this paper is to systematize the dynamical modelisation of all step motors patterns, in order to remove some ambiguities about technology and particularly about drive. The modelisation is made through three main approaches: equations in unmoved coordinates, equations in moving coordinates and finally the dimensionless equations. In this last case, all step actuators offer a single analytical configuration. As the dynamical equations are non linear, a solution but numerical can be given. The physical properties of the different working cases appear clearly : for instance, the step position, or the almost dynamical working and the dynamical working. On the other hand, the effect of the construction parameters on the machine working area is shown. Some of this properties were already taken into account namely in [1, 5-8], with physical models, relating to particular configurations and supplies. This paper should contribute to unify the step motors theory and, moreover, indicate the appropriate coordinates for specific studies.
\end{abstract}

\section{Glossaire des repères utilisés.}

$\alpha, \beta$ : désignent les axes des repères immobiles, liés aux deux plots consécutifs du stator, ou aux deux voies d'enroulement (phase), entourant vant être ilotées sé arément . ainsi, $u_{\alpha}, i_{\alpha}$ sont respectivement les valeurs instantanées de la tension ou du courant dans la voie (phase) $\alpha, L_{\alpha \alpha}$ l'inductance propre de la phase $\alpha, L_{\alpha \beta}$ l'inductance réciproque des phases $\alpha$ et $\beta$, etc...

$\mathrm{a}, \mathrm{b}$ : désignent les composantes orthogonales dans le repère tournant du rotor $; u_{\mathrm{a}}, i_{\mathrm{a}}$ les valeurs instantanées correspondantes de la tension et du courant.

$\mathrm{m} \quad$ : indice du repère mécanique qui correspond à l'angle géométrique, repère qui décrit un cycle mécanique complet (c'est-à-dire qu'après un tour, il revient sur lui-même) parcourt $2 \pi$ radians mécaniques $\left(2 \pi_{\mathrm{m}}\right)$.

e : indice du repère électrique : on appelle, dans ce mémoire, un cycle électrique $=2 \pi$ radians $\pi_{\mathrm{e}}$,

deux pôles magnétiques consécutifs de même nom, situés au rotor (ou entre deux dents consécutives, situées également au rotor); ainsi, un vecteur du champ magnétique considéré revient sur lui-même après avoir décrit $2 \pi$ radians électriques. L'angle électrique est toujours directement proportionnel à l'angle géométrique et constitue un outil mathématique d'emploi commode, permettant de traiter les structures multipolaires (ou multidentaires), à symétrie de révolution, comme des 
structures bipolaires (ou bidentaires) ; la substitution des angles mécaniques réels aux angles électriques se fait toujours au stade final du calcul.

\section{Introduction.}

Nous pouvons distinguer deux principaux types de moteurs pas à pas :

a) les machines à structure rotorique passive, dans lesquelles le rotor est constitué par une couronne ferromagnétique dentée et dont le couple synchronisant « courant du stator-fer »: $C_{\mathrm{em}}^{\mathrm{cf}}$, n'est dû qu'à la variation de la réluctance dans deux axes caractéristiques. Ces machines seront appelées par la suite «à réluctance variable » ou « MRV ».

b) Les machines à structure rotorique active ou polarisée, dans lesquelles le rotor est constitué par plusieurs aimants (pôles), de polarité alternée, situés sur sa périphérie. Ces machines seront appelées « à aimant permanent » ou « MP ». Une autre variante de la machine à rotor actif comporte deux couronnes dentées, décalées, réunies par un aimant permanent central, qui confère aux dents de chaque couronne des polarités différentes ; ainsi, le stator voit autant de pôles magnétiques qu'il y a de dents sur les deux couronnes. Cette machine sera appelée "hybride» ou « $\mathbf{M H}$ ». Les machines à rotor actif fonctionnent grâce au couple synchronisant «courant-aimant » : $C_{\mathrm{em}}^{\mathrm{ca}}$.

En principe, sauf constructions très particulières, les deux couples synchronisants $\left(C_{\mathrm{em}}^{\mathrm{cf}}\right.$ et $\left.C_{\mathrm{em}}^{\mathrm{ca}}\right)$ ne coïncident jamais sur la même machine. Néanmoins, afin d'unifier les analyses dynamiques de toutes les machines pas à pas, nous proposerons un modèle physique qui met en jeu les deux interactions préalablement définies, ainsi que les trois structures géométriques de base (MRV, MP, MH). La séparation des caractéristiques correspondantes sera effectuée au niveau du modèle numérique final.

Au point de vue électrotechnique, le moteur pas à pas ressemble à une machine synchrone, dont le stator (le plus souvent à pôles saillants) porte les enroulements de pilotage et le rotor (toujours à pôles saillants) est soit muni d'un aimant permanent (structure dite " polarisée » ou " active »), soit constitué par une pièce ferromagnétique dentée (structure dite « réluctante» ou « passive »).

La machine synchrone triphasée ( $m=3$, Fig. 1a) est alimentée par le réseau de fréquence, $f$, qui lui impose une fonction de pilotage continue : $\gamma(t)=$ $\omega t=2 \pi f t$ (Fig. 1b), et partant un champ statorique tournant circulaire, à la vitesse électrique constante $\omega$ (ou mécanique $\Omega=\omega / \mathrm{p}$, avec $p$ : nombre de paires de pôles). Dans une machine pas à pas, à 3 voies pilotables séparément (trois phases) (Fig. 2a), l'électronique de puissance et de commande

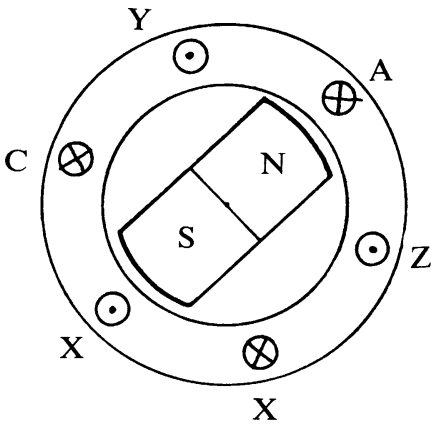

a)

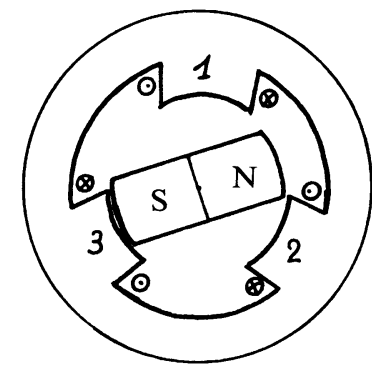

b)
Fig. 1. - Moteur synchrone. Moteur pas à pas.

[Synchronous motor. Step motor.]

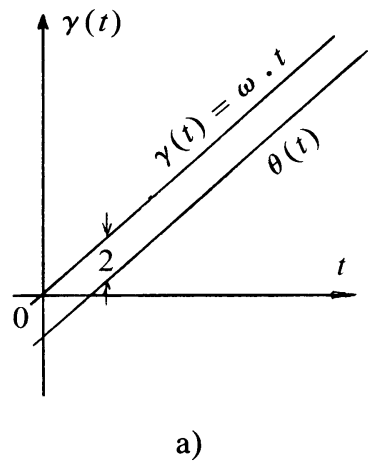

a)

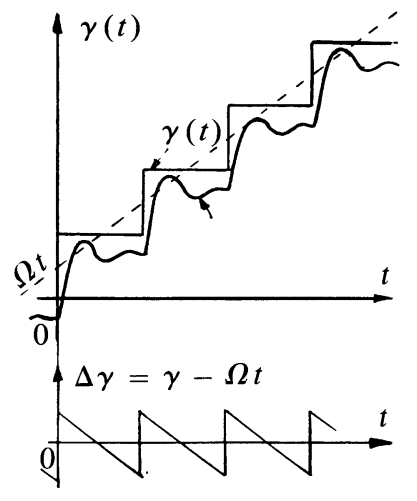

b)
Fig. 2. - Fonction de pilotage du moteur synchrone et du moteur pas à pas.

[Driving function for a synchronous motor and for a step motor.]

entraîne une alimentation séquentielle des différentes bobines du stator. A une étape d'alimentation donnée, correspond une position angulaire électrique de repos du rotor : $\gamma(t)$. La séquence suivante confère à la machine une nouvelle position de repos :

$$
\gamma(t+\mathrm{d} t)=\gamma(t)+\alpha_{\mathrm{pe}}
$$

où : $\alpha_{\mathrm{pe}}$ est un pas angulaire électrique.

La position instantanée du rotor : $\theta_{\mathrm{e}}$, tend vers $\gamma$ avec une évolution qui dépend du couple moteur, de l'inertie, des frottements et du couple de charge. La machine pas à pas ne diffère de la machine synchrone que par la rotation discrète de $\gamma(t)$, que nous appellerons « angle de pilotage».

A la différence de la machine synchrone, la valeur instantanée de la vitesse angulaire de la fonction de 
pilotage n'est pas constante, mais pulsée autour d'une valeur moyenne $\bar{\omega}_{\mathrm{e}}=2 \pi f_{\mathrm{e}}$ (Fig. $2 \mathrm{~b}$ ), avec $f_{\mathrm{e}}$ : fréquence électrique des commutations. Si le déplacement discret de l'angle de pilotage s'opère conformément aux gradins de la figure $2 b$, la droite :

$$
\omega_{\mathrm{e}} t
$$

matérialise la rotation de la première harmonique et la fonction :

$$
\Delta \gamma=\gamma-\omega_{\mathrm{e}} t
$$

mesure la pulsation instantanée de la vitesse angulaire.

Sous réserve de tenir compte de la géométrie particulière et de bien représenter la fonction de pilotage, on doit pouvoir dériver le modèle dynamique de toutes les variantes de la machine pas à pas du modèle similaire de la machine synchrone.

Le passage de la commande au mouvement mécanique du rotor est mené par trois étapes successives :

- la fonction de pilotage $\gamma(t)$, qui constitue une variable indépendante du problème, impose la tension aux différentes voies d'enroulement.

Dans le cas d'une alimentation en tensions, l'évolution temporelle de cette fonction est l'image de la position instantanée du vecteur de la tension $\mathbf{U}$ dans l'axe électrique de chaque voie [2] ;

- les courants statoriques et les champs qu'ils engendrent suivent $\mathbf{U}$ avec un retard dû à la relaxation électromagnétique ;

- l'interaction aimant (ou fer) avec le champ créé par le courant est à l'origine du couple électromagnétique synchronisant, qui met le rotor en mouvement, selon une loi qui ne correspond pas au mouvement du champ à cause des inerties et des pertes tant mécaniques qu'électriques.

Pour une fréquence électrique constante $f_{\mathrm{e}}$ des impulsions de commutation, le mouvement se stabilise et le déplacement discret du vecteur $\mathbf{U}$ (ou du champ statorique $\psi$ ) apparaît comme une oscillation par rapport au rotor, qui s'atténue avec la diminution de $\Delta \gamma$. Dans les raisonnements précédents, nous avons considéré les vitesses et les angles dans le s'appliquent évidemment au repère mécanique.

\section{Equation du mouvement dans un repère immobile.}

Soit le modèle d'une machine pas à pas de la figure 3, qui se compose d'un stator à quatre plots et d'un rotor bipolaire à électro-aimant. Le choix d'un électro-aimant en matériau ferromagnétique permet de prendre en compte à la fois les machines :

- à aimant permanent (MP et $\mathbf{M H}$ ), en remplaçant le courant par une aimantation équivalente et le matériau ferromagnétique par un mobile magnétique avec $\mu_{\mathrm{r}} \cong 1$ et non conducteur ;

- à réluctance variable, en posant le courant rotorique égal à zéro.

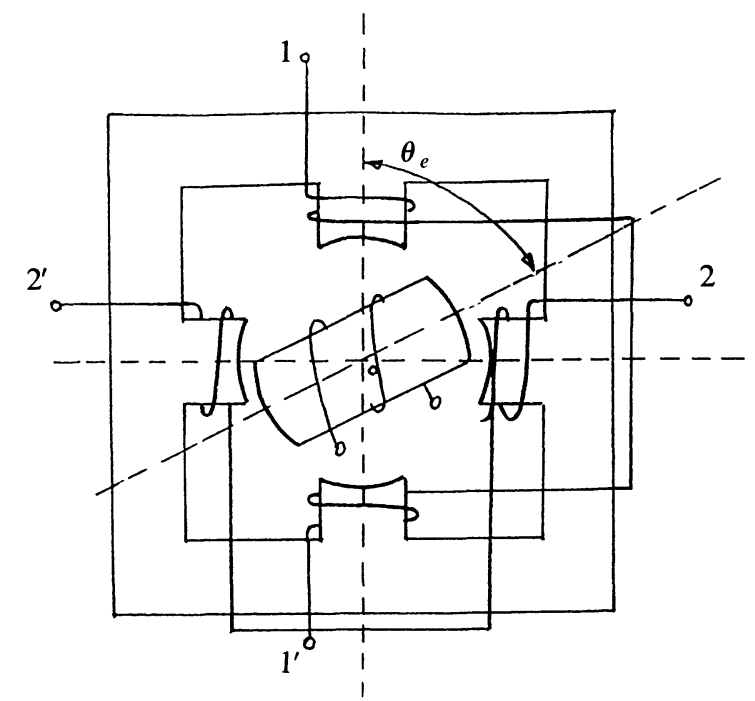

Fig. 3. - Modèle généralisé du moteur pas à pas.

[General model of a step motor.]

Les quatre voies rotoriques sont alimentées deux à deux, selon le cyclogramme $(1,2)(2,-1)(-1,-2)$ $(-2,1)$ mettant en jeu à chaque séquence l'ensemble du cuivre statorique. Ce système d'alimentation constitue le cas le plus général, d'où l'on peut déduire l'alimentation par une seule voie ou par une seule phase.

L'ensemble de la machine est rapporté aux deux axes immobiles $\alpha$ et $\beta$, passant par les plots $11^{\prime}$ et $22^{\prime}$ respectivement. Le rotor bipolaire, où les angles électriques et mécaniques sont confondus, modélise le pas polaire de n'importe quelle machine à structure symétrique (Fig. 4).

$\alpha$

$\theta_{\mathrm{e}}$ 1

$$
\frac{\pi}{p}
$$

Fig. 4. - Configuration d'un pas polaire.

[Configuration of a pole pitch.] 
Le schéma électrique simplifié de la machine bipolaire, ou le schéma d'un pas polaire d'une machine multipolaire, est représenté sur la figure 5.

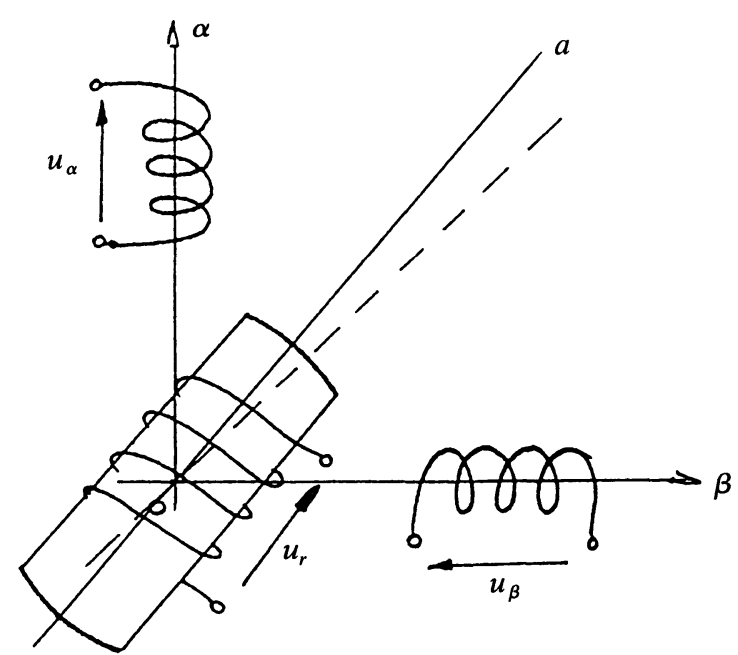

Fig. 5. - Modèle électrique du moteur pas à pas.

[Electrical model of a step motor.]

On supposera, par la suite, que la machine à circuit magnétique simple (single-stack) présente $2 p$ pôles au rotor ou/et $2 p_{z}$ dents ferromagnétiques.

$\mathrm{Si} N_{\mathrm{ps}}$ est le nombre de plots statoriques, $\tau_{\mathrm{s}}=2 \pi_{\mathrm{mec}} / N_{\mathrm{ps}}$ : est l'écart entre deux plots statoriques.

Par ailleurs :

$\theta_{\mathrm{e}}^{\prime} \quad$ : est l'angle électrique pris le long de l'entrefer par rapport à l'origine immobile $\alpha$;

$\theta_{\mathrm{e}}(t)$ : est l'angle électrique de la position instantanée du rotor.

$\gamma(t)$ : est la fonction de pilotage;

$\eta(t)$ : représente l'écart angulaire entre la position du rotor et la position du champ;

$\Omega_{\mathrm{e}} \quad$ : est la vitesse dans le repère électrique.

La liaison entre les grandeurs des repères électrique et mécanique s'écrit, pour le modèle choisi :

$$
\begin{gathered}
\theta_{\mathrm{e}}=\left\{\begin{array}{l}
p \theta_{\mathrm{m}}: \text { pour MP } \\
2 p_{z} \theta_{\mathrm{m}}: \text { pour MRV }
\end{array}\right. \\
\Omega_{\mathrm{e}}=\left\{\begin{array}{l}
p \Omega: \text { pour MP } \\
2 p_{z} \Omega: \text { pour MRV }
\end{array}\right. \\
\Omega=\left\{\begin{array}{l}
\frac{1}{p} \frac{\mathrm{d} \theta_{\mathrm{e}}}{\mathrm{d} t}: \text { pour MP } \\
\frac{1}{2 p_{z}} \frac{\mathrm{d} \theta_{\mathrm{e}}}{\mathrm{d} t}: \text { pour MRV }
\end{array}\right.
\end{gathered}
$$

La machine, modélisée sur la figure 5 , présente théoriquement trois degrés de liberté : deux électriques : tensions (courants) du rotor et du stator, et un mécanique.
Ce qui se traduit par deux équations électriques :

$$
U_{j}=R_{j} i_{j}+\frac{\mathrm{d}}{\mathrm{d} t} \Psi_{j}=R_{j} i_{j}+\frac{\mathrm{d}}{\mathrm{d} t} \sum_{k} L_{j k} i_{k}
$$

avec :

$$
L_{j k}=L_{k j} \quad k \neq j
$$

et une équation mécanique :

$$
C_{\mathrm{r}}=C_{\mathrm{em}}-D_{\mathrm{r} 0} \Omega-J_{0} \frac{\mathrm{d} \Omega}{\mathrm{d} t}-C_{\mathrm{fs} 0}(\operatorname{sign} \Omega)
$$

avec :

$C_{\mathrm{r}}$ : couple résistant

$D_{\mathrm{r} 0}$ : coefficient de frottement visqueux du moteur

$J_{0}$ : inertie du rotor

$C_{\mathrm{fs} 0}$ : couple de frottement sec du moteur

le couple électromagnétique s'exprime classiquement :

$$
C_{\mathrm{em}}=\frac{\partial W_{\mathrm{em}}}{\partial \theta_{\mathrm{m}}}=\frac{1}{2} \sum_{j} \sum_{k} i_{k} i_{j} \frac{\mathrm{d} L_{j k}}{\mathrm{~d} \theta_{\mathrm{m}}}
$$

On peut répartir les forces électromotrices induites en :

- f.e.m. de transformation d'une part :

$$
L \frac{\mathrm{d} i}{\mathrm{~d} t}
$$

— et f.e.m. de rotation d'autre part :

$$
i \frac{\mathrm{d}}{\mathrm{d} t} L=i \frac{\mathrm{d}}{\mathrm{d} \theta_{\mathrm{m}}} L-\frac{\mathrm{d} \theta_{\mathrm{m}}}{\mathrm{d} t}=i M \Omega
$$

où : $M_{i j}=\mathrm{d} L_{i j} / \mathrm{d} \theta_{\mathrm{m}}$ est le mutuel cyclique de Kron.

Les expressions (2) se mettent sous la forme développée :

$$
\begin{aligned}
U_{\alpha}= & R_{\alpha} i_{\alpha}+L_{\alpha \alpha} \frac{\mathrm{d} i_{\alpha}}{\mathrm{d} t}+L_{\alpha \beta} \frac{\mathrm{d} i_{\beta}}{\mathrm{d} t}+ \\
& +L_{\alpha \mathrm{r}} \frac{\mathrm{d} i_{\mathrm{r}}}{\mathrm{d} t}+\left(M_{\alpha \alpha} i_{\alpha}+M_{\alpha \beta} i_{\beta}+M_{\alpha \mathrm{r}} i_{\mathrm{r}}\right) \Omega \\
U_{\beta}= & R_{\beta} i_{\beta}+L_{\alpha \beta} \frac{\mathrm{d} i_{\alpha}}{\mathrm{d} t}+L_{\beta \beta} \frac{\mathrm{d} i_{\beta}}{\mathrm{d} t}+ \\
& +L_{\beta \mathrm{r}} \frac{\mathrm{d} i_{\mathrm{r}}}{\mathrm{d} t}+\left(M_{\alpha \beta} i_{\alpha}+M_{\beta \beta} i_{\beta}+M_{\beta \mathrm{r}} i_{\mathrm{r}}\right) \Omega .
\end{aligned}
$$

Les f.e.m. de rotation sont indépendantes du temps et se calculent à l'aide des schémas électriques équivalents du régime statique. En l'absence de saturation, le système est linéaire.

On peut déduire des figures 4,5 et 6 :

$$
\left[\begin{array}{l}
L_{\alpha \alpha} \\
L_{\beta \beta}
\end{array}\right]=\left[\begin{array}{l}
L_{0} \\
L_{0}
\end{array}\right]+L_{\mathrm{p}}\left[\begin{array}{l}
\cos 2 \theta_{\mathrm{e}} \\
\cos \left(2 \theta_{\mathrm{e}}-2 p_{z} \tau_{\mathrm{s}}\right)
\end{array}\right] .
$$

L'inductance réciproque entre deux voies du stator présente la même variation, en fonction de l'angle $\theta_{\mathrm{e}}\left(\theta_{\mathrm{m}}\right)$, que les inductances propres, mais sa valeur maximale correspond à la position du rotor (ou 
d'une dent rotorique) entre deux plots du stator (Fig. 6). Pour la référence dans l'axe $\alpha$, le développement en séries harmoniques de $L_{\alpha \beta}$ s'écrit :

$$
\begin{aligned}
L_{\alpha \beta}=L_{\alpha \beta 0}+L_{\alpha \beta 2} \cos \left(2 \theta_{\mathrm{e}}-p_{z} \tau_{\mathrm{s}}\right)+ \\
+L_{\alpha \beta 4} \cos \left(4 \theta_{\mathrm{e}}-p_{\mathrm{z}} \tau_{\mathrm{s}}\right) .
\end{aligned}
$$

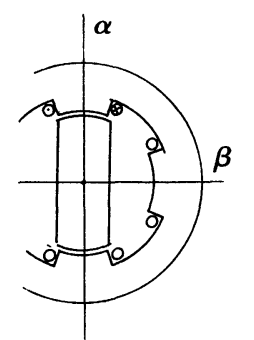

$\phi_{\alpha \beta}=\min$

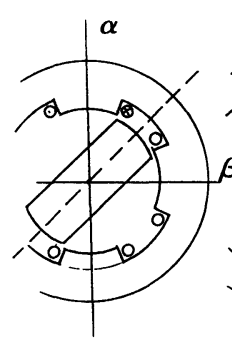

$\phi_{\alpha \beta}=\max$

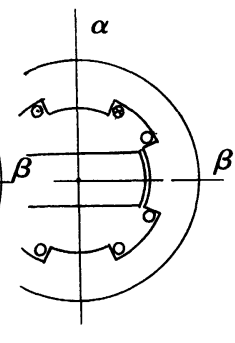

$\phi_{\alpha \beta}=\min$
Fig. 6. - Influence de la position du rotor sur le couplage magnétique entre les phases.

[Influence of the rotor position on the magnetical coupling between windings.]

Par la suite, nous limiterons les calculs aux deux premiers termes de ce développement.

Ainsi :

$$
\begin{aligned}
M_{\alpha \alpha} & =\frac{\mathrm{d}}{\mathrm{d} \theta_{\mathrm{m}}} L_{\alpha \alpha}=-2 p_{z} L_{\mathrm{p}} \sin 2 \theta_{\mathrm{e}} \\
M_{\beta \beta} & =-2 p_{z} L_{\mathrm{p}} \sin 2\left(\theta_{\mathrm{e}}-p_{z} \tau_{\mathrm{s}}\right) \\
M_{\alpha \beta} & =-2 p_{z} L_{\alpha \beta 2} \sin \left(2 \theta_{\mathrm{e}}-p_{z} \tau_{\mathrm{s}}\right) .
\end{aligned}
$$

Par ailleurs :

$$
\begin{aligned}
L_{\alpha \mathrm{r}} & =L_{\alpha \mathrm{r} \max } \cos p \theta_{\mathrm{m}}=L_{\alpha \mathrm{r} \max } \cos \theta_{\mathrm{e}} \\
L_{\beta \mathrm{r}} & =L_{\beta \mathrm{r} \max } \cos p\left(\theta_{\mathrm{m}}-\tau_{\mathrm{s}}\right) \\
& =L_{\beta \mathrm{r} \max } \cos \left(\theta_{\mathrm{e}}-p \tau_{\mathrm{s}}\right) .
\end{aligned}
$$

Soit :

$$
\begin{aligned}
& M_{\alpha \mathrm{r}}=-p L_{\alpha \mathrm{r} \max } \sin p \theta_{\mathrm{m}}=-p L_{\alpha \mathrm{r} \max } \sin \theta_{\mathrm{e}} \\
& M_{\beta \mathrm{r}}=-p L_{\alpha \mathrm{r} \max } \sin \left(\theta_{\mathrm{e}}-p \tau_{\mathrm{s}}\right) .
\end{aligned}
$$

En introduisant (8) dans (7), nous avons :

$$
\begin{aligned}
U_{\alpha}= & R_{\alpha} i_{\alpha}+\left(L_{0}+L_{\mathrm{p}} \cos 2 \theta_{\mathrm{e}}\right) \stackrel{\mathrm{d} i_{\alpha}}{ } \\
+ & {\left[L_{\alpha \beta 0}+L_{\alpha \beta 1} \cos \left(2 \theta_{\mathrm{e}}-p_{z} \tau_{\mathrm{s}}\right)\right] \frac{\mathrm{d} i_{\beta}}{\mathrm{d} t}+e_{\alpha \alpha}+e_{\alpha} } \\
U_{\beta}= & R_{\beta} i_{\beta}+\left[L_{\alpha \beta 0}+L_{\alpha \beta 2} \cos \left(2 \theta_{\mathrm{e}}-p_{z} \tau_{\mathrm{s}}\right)\right] \frac{\mathrm{d} i_{\alpha}}{\mathrm{d} t} \\
& +\left[L_{0}+L_{\mathrm{p}} \cos 2\left(\theta_{\mathrm{e}}-p_{z} \tau_{\mathrm{s}}\right)\right] \frac{\mathrm{d} i_{\beta}}{\mathrm{d} t}+e_{\beta \beta}+e_{\beta}
\end{aligned}
$$

Les f.e.m. de rotation induites, dans une voie d'enroulement, par la variation spatiale des inductances statoriques $\left(e_{\alpha \alpha}, e_{\beta \beta}\right)$ et par la variation spatiale du mutuel "rotor-stator ", s'écrivent, en tenant compte de l'aimant permanent du rotor $\left(i_{\mathrm{r}}=\right.$ Cte $\left.; \mathrm{d} i_{\mathrm{r}} / \mathrm{d} t \equiv 0\right)$, comme :

$$
\begin{aligned}
e_{\alpha \alpha}= & \left(M_{\alpha \alpha} i_{\alpha}+M_{\alpha \beta} i_{\beta}\right) \Omega \\
= & -2 p_{z} \Omega\left[i_{\alpha} L_{\mathrm{p}} \sin 2 \theta_{\mathrm{e}}+\right. \\
& \left.+i_{\beta} L_{\alpha \beta 2} \sin \left(2 \theta_{\mathrm{e}}-p_{z} \tau_{\mathrm{s}}\right)\right] \\
e_{\alpha}= & M_{\alpha \mathrm{r}} i_{\mathrm{r}} \Omega=-p L_{\alpha \mathrm{r} \max } i_{\mathrm{r}} \Omega \sin \theta_{\mathrm{e}} \\
= & -p \psi_{\max } \Omega \sin \theta_{\mathrm{e}}=-K_{\phi} \Omega \sin \theta_{\mathrm{e}} \\
e_{\beta \beta}= & \left(M_{\alpha \beta} i_{\alpha}+M_{\beta \beta} i_{\beta}\right) \Omega \\
= & -2 p_{z} \Omega\left[i_{\alpha} L_{\alpha \beta 2} \sin \left(2 \theta_{\mathrm{e}}-p_{z} \tau_{\mathrm{s}}\right)\right. \\
& \left.+i_{\beta} L_{p} \sin 2\left(\theta_{\mathrm{e}}-p_{z} \tau_{\mathrm{s}}\right)\right] \\
e_{\beta}= & M_{\beta r} i_{r} \Omega=-K_{\phi} \Omega \sin \left(\theta_{\mathrm{e}}-p \tau_{\mathrm{s}}\right) .
\end{aligned}
$$

Le couple électromagnétique, développé par la machine, se déduit le plus aisément de la formule (4) :

$$
\begin{aligned}
C_{\mathrm{em}}= & C_{\mathrm{em}}^{\mathrm{cf}}+C_{\mathrm{em}}^{\mathrm{ca}}=\frac{1}{2} i_{\alpha}^{2} \frac{\mathrm{d} L_{\alpha \alpha}}{\mathrm{d} \theta_{\mathrm{m}}}+\frac{1}{2} i_{\beta}^{2} \frac{\mathrm{d} L_{\beta \beta}}{\mathrm{d} \theta_{\mathrm{m}}} \\
+ & i_{\alpha} i_{\beta} \frac{\mathrm{d} L_{\alpha \beta}}{\mathrm{d} \theta_{\mathrm{m}}}+i_{\mathrm{r}} i_{\alpha} \frac{\mathrm{d} L_{\alpha \mathrm{r}}}{\mathrm{d} \theta_{\mathrm{m}}}+i_{\mathrm{r}} i_{\beta} \frac{\mathrm{d} L_{\beta \mathrm{r}}}{\mathrm{d} \theta_{\mathrm{m}}} \\
= & -p_{z}\left\{L_{\mathrm{p}}\left[i_{\alpha}^{2} \sin 2 \theta_{\mathrm{e}}+i_{\beta}^{2} \sin 2\left(\theta_{\mathrm{e}}-p_{z} \tau_{\mathrm{s}}\right)\right]\right. \\
& \left.+2 i_{\alpha} i_{\beta} L_{\alpha \beta 2} \sin \left(2 \theta_{\mathrm{e}}-p_{z} \tau_{\mathrm{s}}\right)\right\} \\
& -K_{\phi}\left[i_{\alpha} \sin \theta_{\mathrm{e}}+i_{\beta} \sin \left(\theta_{\mathrm{e}}-p \tau_{\mathrm{s}}\right)\right]
\end{aligned}
$$

Les expressions (9) et (10) décrivent la dynamique de la machine à deux voies (phases) commutables en même temps. Pour une machine commutable voie par voie, il suffit de poser $\tau_{\mathrm{s}}=0$.

Montrons l'application pratique des équations du mouvement à la configuration de la figure 3 , qui se caractérise par :

$$
\tau_{\mathrm{s}}=\frac{2 \pi}{N_{\mathrm{ps}}}=\frac{\pi}{2} \quad \text { et } \quad \frac{\pi}{p}=\frac{\pi}{p_{z}}=\frac{2 \pi}{N_{\mathrm{dr}}}=\pi .
$$

Dans ce cas :

$L_{\alpha \alpha}=L_{0}+L_{\mathrm{p}} \cos 2 \theta_{\mathrm{e}} ; \quad L_{\beta \beta}=L_{0}-L_{\mathrm{p}} \cos 2 \theta_{\mathrm{e}}$.

L'inductance réciproque entre deux voies du stator :

$$
{ }_{\alpha \beta}={ }_{\alpha \beta 0} \quad{ }_{\alpha \beta 2} \cos \quad \mathrm{e}-p_{z} \overline{2}
$$

où $L_{\alpha \beta}$ peut être approchée par la formule :

$$
L_{\alpha \beta} \approx L_{\alpha \beta 2} \cos \left(2 \theta_{\mathrm{e}}-\frac{\pi}{2}\right)=L_{\mathrm{p}} \sin 2 \theta_{\mathrm{e}}
$$

car les axes magnétiques des enroulements concernés, décalés dans l'espace de $\pi / 2$ radian mécanique, sont en quadrature électrique : $\theta_{\mathrm{e}}=2 p_{z}$ $\theta_{\mathrm{m}}=2 \pi / 2=\pi$ rad électr. (c'est-à-dire entièrement couplés), pour les f.e.m. de transformation induites par leurs flux réciproques. La figure 6 
illustre bien ce phénomène. Ainsi, lorsqu'une dent du rotor se positionne en face d'un plot du stator, on peut considérer que $\phi_{\alpha \beta}=\min$; par contre, le flux est maximal pour $\theta_{\mathrm{m}}=\pi / 4$; or, dans cette position, la partie spatialement variable de l'inductance propre de l'enroulement (dont le flux est fermé par un circuit magnétique pratiquement identique à $\phi_{\alpha \beta}$ ) correspond à $L_{\mathrm{p}}$. Remarquons que certains auteurs étudiant les machines synchrones proposent de mettre $L_{\alpha \beta}$ sous la forme d'une inductance négative :

$$
L_{\alpha \beta}=-\left[L_{\alpha \beta 0}+L_{\alpha \beta 2} \cos \left(2 p_{z} \theta_{\mathrm{m}}-p_{z} \tau_{\mathrm{s}}\right)\right]
$$

car les axes des enroulements couplés par $\phi_{\alpha \beta}$ sont généralement décalés d'un angle supérieur à $\pi / 2$, et le courant "positif» dans un enroulement induit le courant "négatif » dans l'autre.

En ce qui nous concerne, nous ne voyons pas l'utilité d'une telle formulation pour les machines pas à pas. Par ailleurs, l'action de $L_{\alpha \beta 0}$, pratiquement négligeable, ne peut concerner que la f.e.m. de transformation et n'apporte rien au point de vue physique dans les raisonnements qui vont suivre.

Les inductances « enroulement-aimant " s'écrivent simplement :

$$
\left[\begin{array}{l}
L_{\alpha \mathrm{r}} \\
L_{\beta \mathrm{r}}
\end{array}\right]=L_{\alpha \mathrm{r} \max }\left[\begin{array}{c}
\cos \theta_{\mathrm{e}} \\
\sin \theta_{\mathrm{e}}
\end{array}\right]
$$

ce qui donne finalement :

$$
\begin{aligned}
U_{\alpha}=R i_{\alpha} & +\left(L_{0}+L_{\mathrm{p}} \cos 2 \theta_{\mathrm{e}}\right) \frac{\mathrm{d} i_{\alpha}}{\mathrm{d} t}+ \\
& +L_{\mathrm{p}} \sin 2 \theta_{\mathrm{e}} \frac{\mathrm{d} i_{\beta}}{\mathrm{d} t}-2 K_{\mathrm{r}}\left(i_{\alpha} \sin 2 \theta_{\mathrm{e}}\right. \\
& \left.-i_{\beta} \cos 2 \theta_{\mathrm{e}}\right) \Omega-K_{\phi} \sin \theta_{\mathrm{e}} \Omega
\end{aligned}
$$

$U_{\beta}=R i_{\beta}+L_{\mathrm{p}} \sin 2 \theta_{\mathrm{e}} \frac{\mathrm{d} i_{\alpha}}{\mathrm{d} t}+\left(L_{0}-L_{p} \cos 2 \theta_{\mathrm{e}}\right) \frac{\mathrm{d} i_{\beta}}{\mathrm{d} t}$

$+2 K_{\mathrm{r}}\left(i_{\alpha} \cos 2 \theta_{\mathrm{e}}+i_{\beta} \sin 2 \theta_{\mathrm{e}}\right) \Omega+K_{\phi} \cos \theta_{\mathrm{e}} \Omega$.

Le couple électromagnétique se calcule conformément à (10) :

$$
\begin{aligned}
& C_{\mathrm{em}}^{\mathrm{ca}}=-K_{\phi}\left(i_{\alpha} \sin \theta_{\mathrm{e}}-i_{\beta} \cos \theta_{\mathrm{e}}\right) \\
& C_{\mathrm{em}}^{\mathrm{cf}}=-K_{\mathrm{r}}\left[\left(i_{\alpha}^{2}-i_{\beta}^{2}\right) \sin 2 \theta_{\mathrm{e}}-2 i_{\alpha} i_{\beta} \cos 2 \theta_{\mathrm{e}}\right] .
\end{aligned}
$$

Rappelons que la constante du flux est définie classiquement :

$$
K_{\phi}=p L_{\alpha \mathrm{r} \max } i_{\mathrm{r}}=p \psi_{\mathrm{m}}: \text { pour MP }
$$

et la constante de la réluctance est égale à :

$$
K_{\mathrm{r}}=p_{z} L_{\mathrm{p}}=\frac{N_{\mathrm{dr}}}{2} L_{\mathrm{p}}: \text { pour MRV . }
$$

Pour un modèle hypothétique de la machine hybride, où les deux couples (13) et (14) peuvent être développés :

$$
\begin{gathered}
K_{\phi}=p \psi_{\mathrm{m}}=N_{\mathrm{dr}} \psi_{\mathrm{m}}=p_{z} \psi_{\mathrm{m}} \\
K_{\mathrm{r}}=p_{z} L_{\mathrm{p}}=N_{\mathrm{dr}} L_{\mathrm{p}}=p L_{\mathrm{p}}
\end{gathered}
$$

avec: $N_{\mathrm{dr}}$ : nombre de dents sur une couronne rotorique.

Remarquons qu'une telle machine peut être constituée par deux couronnes rotoriques de la figure 3 , décalées dans l'espace d'un demi-pas dentaire.

Les expressions (11), (12), (13) et (14) définissent pleinement le comportement dynamique des moteurs pas à pas. Les équations correspondantes étant non linéaires, la solution générale ne peut être obtenue que sous une forme numérique. La programmation correspondante s'effectue plus facilement par un changement de repère et le passage à des grandeurs adimensionnelles.

\section{Equation du mouvement dans un repère mobile.}

La résolution numérique des expressions (11), (12), (13) et (14), est beaucoup plus commode dans un repère mobile, qui fait disparaître le produit des variables et des fonctions trigonométriques de la position du rotor.

En prenant comme référence l'axe a du rotor (vecteur du champ tournant rotorique pour une machine à aimant), tournant à la vitesse mécanique $\Omega$, défini dans le repère électrique par (1), la transformation des tensions $u_{\alpha}, u_{\beta}$, s'effectue classiquement à l'aide de la figure 7 :

$$
\begin{gathered}
{\left[\begin{array}{l}
u_{\alpha} \\
u_{\beta}
\end{array}\right]=\left[\begin{array}{cc}
\cos \theta_{\mathrm{e}}-\sin \theta_{\mathrm{e}} \\
\sin \theta_{\mathrm{e}} & \cos \theta_{\mathrm{e}}
\end{array}\right]\left[\begin{array}{l}
u_{\mathrm{a}} \\
u_{\mathrm{b}}
\end{array}\right]} \\
{\left[\begin{array}{l}
u_{\mathrm{a}} \\
u_{\mathrm{b}}
\end{array}\right]=\left[\begin{array}{rr}
\cos \theta_{\mathrm{e}} \sin \theta_{\mathrm{e}} \\
-\sin \theta_{\mathrm{e}} & \cos \theta_{\mathrm{e}}
\end{array}\right]\left[\begin{array}{l}
u_{\alpha} \\
u_{\beta}
\end{array}\right] .}
\end{gathered}
$$

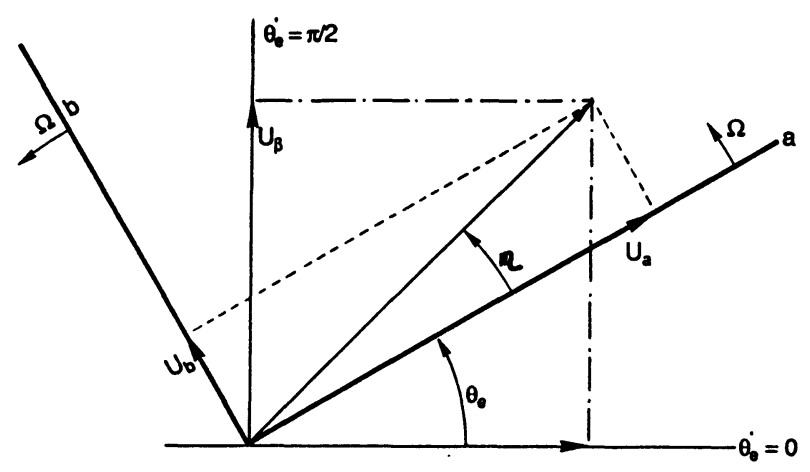

Fig. 7. - Diagramme vectoriel du moteur pas à pas.

[Vectorial diagram of a step motor.] 
Comme les puissances instantanées dans les deux systèmes sont identiques: $p=u_{\alpha} i_{\alpha}+u_{\beta} i_{\beta}=$ $u_{\mathrm{a}} i_{\mathrm{a}}+u_{\mathrm{b}} i_{\mathrm{b}}$ :

$$
\left[\begin{array}{c}
i_{\alpha} \\
i_{\beta}
\end{array}\right]=\left[\begin{array}{lr}
\cos \theta_{\mathrm{e}}-\sin \theta_{\mathrm{e}} \\
\sin \theta_{\mathrm{e}} & \cos \theta_{\mathrm{e}}
\end{array}\right]\left[\begin{array}{c}
i_{\mathrm{a}} \\
i_{\mathrm{b}}
\end{array}\right]
$$

et :

$$
\left[\begin{array}{c}
i_{\mathrm{a}} \\
i_{\mathrm{b}}
\end{array}\right]=\left[\begin{array}{rr}
\cos \theta_{\mathrm{e}} & \sin \theta_{\mathrm{e}} \\
-\sin \theta_{\mathrm{e}} & \cos \theta_{\mathrm{e}}
\end{array}\right]\left[\begin{array}{c}
i_{\alpha} \\
i_{\beta}
\end{array}\right] .
$$

Remarque importante. On doit, en principe, dérouler les calculs, qui vont suivre, séparément pour les machines MP et MRV, car, d'une part, la fréquence spatiale électrique n'est pas la même dans ces deux dispositifs, et, d'autre part, leurs couples, de nature différente, n'agissent pas simultanément dans les constructions classiques. Néanmoins, en vue de l'écriture d'un programme numérique commun, nous préférons traiter les expressions (11), (12), (13) et (14), d'un seul bloc. Dans ces conditions, on va considérer implicitement le modèle d'une $\mathbf{M H}$, dont la constante de flux est définie par (15) et la constante de la réluctance par (16).

Compte tenu de (17), (18), (19) et (20) :

$$
\begin{aligned}
\frac{\mathrm{d} i_{\mathrm{a}}}{\mathrm{d} t}= & \frac{\mathrm{d}}{\mathrm{d} t}\left(i_{\alpha} \cos \theta_{\mathrm{e}}+i_{\beta} \sin \theta_{\mathrm{e}}\right) \\
= & \frac{\mathrm{d} i_{\alpha}}{\mathrm{d} t} \cos \theta_{\mathrm{e}}+\frac{\mathrm{d} i_{\beta}}{\mathrm{d} t} \sin \theta_{\mathrm{e}} \\
& +\left(-i_{\alpha} \sin \theta_{\mathrm{e}}+i_{\beta} \cos \theta_{\mathrm{e}}\right) \frac{\mathrm{d} \theta_{\mathrm{e}}}{\mathrm{d} t}
\end{aligned}
$$

soit :

$$
\frac{\mathrm{d} i_{\alpha}}{\mathrm{d} t} \cos \theta_{\mathrm{e}}+\frac{\mathrm{d} i_{\beta}}{\mathrm{d} t} \sin \theta_{\mathrm{e}}=\frac{\mathrm{d} i_{\mathrm{a}}}{\mathrm{d} t}-i_{\mathrm{b}} \frac{\mathrm{d} \theta_{\mathrm{e}}}{\mathrm{d} t}
$$

et :

$$
\begin{aligned}
\frac{\mathrm{d} i_{\mathrm{b}}}{\mathrm{d} t}=\left(-\frac{\mathrm{d} i_{\alpha}}{\mathrm{d} t} \sin \theta_{\mathrm{e}}\right. & \left.+\frac{\mathrm{d} i_{\beta}}{\mathrm{d} t} \cos \theta_{\mathrm{e}}\right) \\
& -\left(i_{\alpha} \cos \theta_{\mathrm{e}}+i_{\beta} \sin \theta_{\mathrm{e}}\right) \frac{\mathrm{d} \theta_{\mathrm{e}}}{\mathrm{d} t}
\end{aligned}
$$

donc :

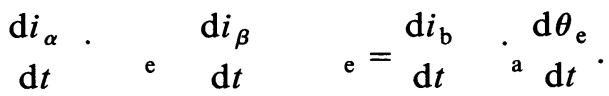

En multipliant maintenant l'expression (11) par $\cos \theta_{\mathrm{e}}$ et l'expression (12) par $\sin \theta_{\mathrm{e}}$, on obtient, après la sommation :

$$
\begin{aligned}
U_{\mathrm{a}}= & R i_{\mathrm{a}}+\left(L_{0}+L_{\mathrm{p}}\right)\left(\frac{\mathrm{d} i_{\alpha}}{\mathrm{d} t} \cos \theta_{\mathrm{e}}+\frac{\mathrm{d} i_{\beta}}{\mathrm{d} t} \sin \theta_{\mathrm{e}}\right) \\
& +2 K_{\mathrm{r}} i_{\mathrm{b}} \Omega \\
= & R i_{\mathrm{a}}+\left(L_{0}+L_{\mathrm{p}}\right)\left(\frac{\mathrm{d} i_{\mathrm{a}}}{\mathrm{d} t}-i_{\mathrm{b}} \frac{\mathrm{d} \theta_{\mathrm{e}}}{\mathrm{d} t}\right) \\
& +2 p_{z} L_{\mathrm{p}} i_{\mathrm{b}} \Omega .
\end{aligned}
$$

De même, en multipliant l'expression (11) par $-\sin \theta_{\mathrm{e}}$ et l'expression (12) par $\cos \theta_{\mathrm{e}}$, nous obtenons :

$$
\begin{aligned}
U_{\mathrm{b}}= & R i_{\mathrm{b}}+\left(L_{0}-L_{\mathrm{p}}\right)\left(-\frac{\mathrm{d} i_{\alpha}}{\mathrm{d} t} \sin \theta_{\mathrm{e}}+\frac{\mathrm{d} i_{\beta}}{\mathrm{d} t} \cos \theta_{\mathrm{e}}\right) \\
& +2 K_{\mathrm{r}} i_{\mathrm{a}} \Omega+K_{\phi} \Omega \\
= & R i_{\mathrm{b}}+\left(L_{0}-L_{\mathrm{p}}\right)\left(\frac{\mathrm{d} i_{\mathrm{b}}}{\mathrm{d} t}+i_{\mathrm{a}} \frac{\mathrm{d} \theta_{\mathrm{e}}}{\mathrm{d} t}\right) \\
& +2 p_{z} L_{\mathrm{p}} i_{\mathrm{a}} \Omega+K_{\phi} \Omega .
\end{aligned}
$$

L'expression du couple électromagnétique (14) et (15) s'écrit dans les coordonnées mobiles :

$$
C_{\mathrm{em}}=C_{\mathrm{em}}^{\mathrm{ca}}+C_{\mathrm{em}}^{\mathrm{cf}}=K_{\phi} i_{\mathrm{b}}+2 K_{\mathrm{r}} i_{\mathrm{a}} i_{\mathrm{b}} .
$$

D'où l'équation complète du mouvement mécanique :

$$
\begin{aligned}
& C_{\mathrm{em}}=C_{\mathrm{r}}+J_{0} \frac{\mathrm{d} \Omega}{\mathrm{d} t}+C_{\mathrm{fs} 0} \operatorname{sign}(\Omega)= \\
&=K_{\phi} i_{\mathrm{b}}+2 K_{\mathrm{r}} i_{\mathrm{a}} i_{\mathrm{b}} .
\end{aligned}
$$

Soit en regroupant les équations du mouvement :

$$
\begin{gathered}
U_{a}=R i_{\mathrm{a}}+\left(L_{0}+L_{\mathrm{p}}\right) \frac{\mathrm{d} i_{\mathrm{a}}}{\mathrm{d} t}-p\left(L_{0}-L_{\mathrm{p}}\right) i_{\mathrm{b}} \Omega \\
U_{\mathrm{b}}=p\left(L_{0}+L_{\mathrm{p}}\right) \Omega i_{\mathrm{a}}+R i_{\mathrm{b}}+ \\
+\left(L_{0}-L_{\mathrm{p}}\right) \frac{\mathrm{d} i_{\mathrm{b}}}{\mathrm{d} t}+K_{\phi} \Omega \\
C_{\mathrm{em}}=2 K_{\mathrm{r}} i_{\mathrm{a}} i_{\mathrm{b}}+K_{\phi} i_{\mathrm{b}}= \\
=C_{\mathrm{r}}+J_{0} \frac{\mathrm{d} \Omega}{\mathrm{d} t}+D_{\mathrm{r} 0} \Omega+C_{\mathrm{fs} 0} \operatorname{sign}(\Omega) .
\end{gathered}
$$

On peut remarquer aisément que le système (25), quoiqu'établi pour une machine hybride, alimentée par deux tensions à la fois, peut servir moyennant les initialisations appropriées et l'introduction de paramètres différents pour $K_{\mathrm{r}}$ et $K_{\phi}$ à la modélisation de n'importe quelle machine à répartition régulière des plots et des dents (pôles). Cette machine peut être alimentée en tension (par une ou plusieurs voies) ou en courant. Le chapitre 4 va illustrer ces propos sur un exemple d'initialisation concrète.

Le système (25) permet de calculer numériquement $i t$ et $t$ et partant es caracteristiques $\mathrm{u}$ fonctionnement dynamique $C_{\mathrm{em}}(\Omega), C_{\mathrm{em}}(t)$, pour une charge $C_{\mathrm{r}}$ donnée. Les paramètres $L_{0}, L_{\mathrm{p}}$, $K_{\phi}, K_{\mathrm{r}}, D_{\mathrm{r} 0}, C_{\mathrm{fs} 0}$, peuvent être mesurés ou recherchés dans les catalogues des constructeurs.

Pour faciliter l'écriture des programmes qui englobent le plus grand nombre de machines, il est souhaitable de mettre (25) sous une forme adimensionnelle, en rapportant tout d'abord les tensions à la tension résultante d'alimentation. Pour une alimentation simultanée des deux phases avec la tension U (Fig. 7) : 


$$
\begin{gathered}
\underline{u_{\mathrm{a}}}=\frac{u_{\mathrm{a}}}{\sqrt{2} U}=\frac{\sqrt{2} U \cos \eta}{\sqrt{2}}=\cos \eta \\
\underline{u_{\mathrm{b}}}=\frac{u_{\mathrm{b}}}{\sqrt{2} U}=\sin \eta \\
\underline{i_{\mathrm{a}}}=\frac{i_{\mathrm{a}}}{\sqrt{2} \frac{U}{R}}=\frac{i_{\mathrm{a}}}{\sqrt{2} I} \\
\underline{i_{\mathrm{b}}}=\frac{i_{\mathrm{b}}}{\sqrt{2} \frac{U}{R}}=\frac{i_{\mathrm{b}}}{\sqrt{2} I} .
\end{gathered}
$$

Par la suite, il est souhaitable $\left(^{1}\right)$, pour la clarté des calculs, de séparer les machines à aimants (MP MH) des machines à réluctance variable (MRV).

3.1 MAChines A AIMANT $\left(L_{\mathrm{p}}=0\right.$, DONC $\left.K_{\mathrm{r}}=0\right)$. - Le temps de base peut correspondre à la période des oscillations libres $T_{\phi}$, soit pour une MP selon [3] :

$$
\begin{gathered}
T_{\phi}=\frac{1}{\omega_{\phi}} \\
T_{\phi}^{-1}=\sqrt{\frac{C_{\mathrm{max}}}{J_{0}}}=\sqrt{\frac{p C_{\mathrm{em} \max }^{\mathrm{ca}}}{J_{0}}}
\end{gathered}
$$

d'où le temps relatif :

$$
\begin{gathered}
\underline{t}=\frac{t}{T_{\phi}}=\omega_{\Phi} t \rightarrow \underline{t}=\omega_{\Phi}^{-1} t \rightarrow \frac{\mathrm{d}}{\mathrm{d} \underline{t}}=\frac{\mathrm{d}}{\mathrm{d}\left(\omega_{\Phi}^{-1} t\right)} \\
\frac{\mathrm{d}}{\mathrm{d} \underline{t}}=\omega_{\Phi} \frac{\mathrm{d}}{\mathrm{d} t} .
\end{gathered}
$$

En divisant (21) et (22) par $\sqrt{2} U$, on obtient :

$$
\frac{u_{\mathrm{a}}}{\sqrt{2} U}=\frac{R i_{\mathrm{a}}}{\sqrt{2} U}+\frac{L_{0}}{\sqrt{2} U} \frac{\mathrm{d} i_{\mathrm{a}}}{\mathrm{d} t}-\frac{L_{0}}{\sqrt{2} U} p i_{\mathrm{b}} \Omega
$$

soit :

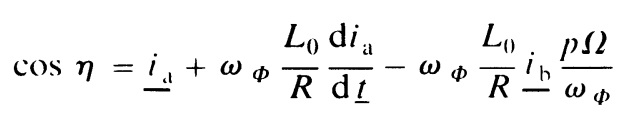

comme :

$$
\Omega=\frac{\mathrm{d} \theta_{\mathrm{m}}}{\mathrm{d} t}=\frac{1}{p} \frac{\mathrm{d} \theta_{\mathrm{e}}}{\mathrm{d} t}
$$

d'où :

$$
\omega_{\Phi} \frac{L_{0}}{R} i_{\mathrm{b}} \frac{1}{p} \frac{\mathrm{d} \theta_{\mathrm{e}}}{\mathrm{d} t} \cdot \frac{p}{\omega_{\Phi}}=\omega_{\Phi} \frac{L_{0}}{R} i_{\mathrm{b}} \frac{\mathrm{d} \theta_{\mathrm{e}}}{\mathrm{d} \underline{t}}
$$

(') Mais pas nécessaire comme va le montrer le résultat final. et finalement :

$$
\left.\begin{array}{c}
\cos \eta=\underline{i}_{\mathrm{a}}+\omega_{\Phi} \frac{L_{0}}{R} \frac{\mathrm{d} \underline{i}_{\mathrm{a}}}{\mathrm{d} \underline{t}}-\omega_{\Phi} \frac{L_{0}}{R} \underline{i_{\mathrm{b}}} \frac{\mathrm{d} \theta_{\mathrm{e}}}{\mathrm{d} \underline{t}} \\
\text { ou encore } \\
\cos \eta=\underline{i_{\mathrm{a}}}+\underline{T_{\mathrm{e} 0}} \frac{\mathrm{d} i_{\mathrm{a}}}{\mathrm{d} t}-\underline{T_{\mathrm{e} 0}} \frac{i_{\mathrm{b}}}{\mathrm{d} \theta_{\mathrm{e}}} \frac{\mathrm{d}_{\underline{t}}}{\text { avec }}=\underline{T_{\mathrm{e} 0}}=\frac{\omega_{\Phi}}{\frac{L_{0}}{R}}
\end{array}\right\}
$$

de même :

$$
\begin{aligned}
\sin \eta=i_{\mathrm{b}}+\omega_{\Phi} \frac{L_{0}}{R} \frac{\mathrm{d} i_{\mathrm{b}}}{\mathrm{d} \underline{t}}+\omega_{\Phi} \frac{L_{0}}{R} & \underline{i_{\mathrm{a}}} \frac{\mathrm{d} \theta_{\mathrm{e}}}{\mathrm{d} \underline{t}} \\
& +\frac{1}{p} K_{\Phi} \frac{\mathrm{d} \theta_{\mathrm{e}}}{\mathrm{d} t} / \sqrt{2} U
\end{aligned}
$$

comme :

$$
\frac{1}{p} K_{\Phi} \frac{\mathrm{d} \theta_{\mathrm{e}}}{\mathrm{d} t} / \sqrt{2} U=\frac{\omega_{\Phi} K_{\Phi}}{p \sqrt{2} U} \frac{\mathrm{d} \theta_{\mathrm{e}}}{\mathrm{d} t}
$$

et :

$$
\frac{\omega_{\Phi} K_{\Phi}}{p \sqrt{2} U}=\frac{1}{\omega_{\Phi}} \cdot \frac{1}{\frac{J_{0} R}{K_{\Phi}^{2}}}=\left(\omega_{\Phi} T_{\mathrm{em} 0}\right)^{-1}=\underline{T_{\mathrm{em} 0}^{-1}}
$$

avec $T_{\mathrm{em} 0}=\frac{J_{0} R}{K_{\Phi}^{2}}$ constante de temps électromécanique de l'actionneur.

Nous avons finalement pour la deuxième équation électrique :

$$
\sin \eta=\underline{i_{\mathrm{b}}}+\underline{T_{\mathrm{e} 0}} \frac{\mathrm{d} i_{\mathrm{b}}}{\mathrm{d} \underline{t}}+\left(\underline{T_{\mathrm{e} 0}} \underline{i_{\mathrm{a}}}+\underline{T_{\mathrm{em} 0}^{-1}}\right) \frac{\mathrm{d} \theta_{\mathrm{e}}}{\mathrm{d} \underline{t}} .
$$

La charge se compose dans un cas général:

- d'un élément inertiel : $J_{\text {ch }}$;

- d'un élément dissipatif, fonction de la vitesse : $D_{\text {ch }}$;

- d'un élément dissipatif, fonction du sens de déplacement (frottement sec, amortissement) : $C_{\text {fsch }}$.

En posant :

$$
\begin{gathered}
J_{\mathrm{r}}=J_{0}+J_{\mathrm{ch}} ; \quad D_{\mathrm{r}}=D_{\mathrm{r} 0}+D_{\mathrm{ch}}\left({ }^{2}\right) ; \\
C_{\mathrm{fsr}}=C_{\mathrm{fs} 0}+C_{\mathrm{fsch}}
\end{gathered}
$$

(2) On peut considérer que la charge dissipative, $D_{\text {ro }}$, est du type interne à la machine et qu'elle englobe non seulement les frottements visqueux, mais également les dissipations électriques, fonctions de la vitesse, c'est-à-dire une partie des pertes fer au moins. 
l'expression (24) se met sous la forme :

$$
\begin{aligned}
C_{\mathrm{em}}=\left(J_{0}+J_{\mathrm{ch}}\right) \frac{\mathrm{d} \Omega}{\mathrm{d} t} & +\left(D_{\mathrm{r} 0}+D_{\mathrm{ch}}\right) \Omega \\
& +\left(C_{\mathrm{fs} 0}+C_{\mathrm{fsch}}\right)(\operatorname{sign} \Omega)
\end{aligned}
$$

en divisant par $J_{\mathrm{r}}$, on obtient :

$$
\frac{C_{\mathrm{em}}}{J_{\mathrm{r}}}=\frac{\mathrm{d} \Omega}{\mathrm{d} t}+\frac{D_{\mathrm{r}}}{J_{\mathrm{r}}} \Omega+\frac{C_{\mathrm{fsr}}(\operatorname{sign} \Omega)}{J_{\mathrm{r}}}=K_{\Phi} \cdot \frac{i_{\mathrm{b}}}{J_{\mathrm{r}}}
$$

comme :

$$
\begin{gathered}
\frac{\mathrm{d} \Omega}{\mathrm{d} t}=\frac{\mathrm{d}^{2} \theta_{\mathrm{m}}}{\mathrm{d} t^{2}}=\frac{1}{p} \frac{\mathrm{d}^{2} \theta_{\mathrm{e}}}{\mathrm{d} t^{2}}=\frac{\omega_{\Phi}^{2}}{p} \frac{\mathrm{d}^{2} \theta_{\mathrm{e}}}{\mathrm{d} \underline{t}^{2}} \\
\frac{D_{\mathrm{r}}}{J_{\mathrm{r}}} \Omega=\frac{D_{\mathrm{r}} \omega_{\Phi}}{p J_{\mathrm{r}}} \frac{\mathrm{d} \theta_{\mathrm{e}}}{\mathrm{d} \underline{t}} \\
\frac{K_{\Phi} i_{\mathrm{b}}}{J_{\mathrm{r}}}=\frac{K_{\Phi} \sqrt{2} U}{J_{\mathrm{r}} \frac{\sqrt{2} U}{R}} i_{\mathrm{b}}=\frac{K_{\Phi} \sqrt{2} U}{R J_{\mathrm{r}}} i_{\mathrm{b}}
\end{gathered}
$$

en multipliant par $p / \omega_{\phi}^{2}$, nous avons :

$$
\begin{aligned}
\frac{\mathrm{d}^{2} \theta_{\mathrm{e}}}{\mathrm{d} \underline{t}^{2}}+\frac{D_{\mathrm{r}}}{J_{\mathrm{r}} \omega_{\Phi}} & \frac{\mathrm{d} \theta_{\mathrm{e}}}{\mathrm{d} \underline{t}}+\frac{p}{J_{\mathrm{r}} \omega_{\Phi}^{2}} C_{\mathrm{fsr}} \\
& \times \operatorname{sign}\left(\frac{\mathrm{d} \theta_{\mathrm{e}}}{\mathrm{d} t}\right)=\frac{p K_{\Phi} \sqrt{2} U}{J_{\mathrm{r}} \omega_{\Phi}^{2} R} i_{\mathrm{b}}
\end{aligned}
$$

ou sous une forme adimensionnelle :

$$
\begin{aligned}
& \frac{\mathrm{d}^{2} \theta_{\mathrm{e}}}{\mathrm{d} \underline{t}^{2}}+\underline{T_{\mathrm{mr}}^{-1}} \frac{\mathrm{d} \theta_{\mathrm{e}}}{\mathrm{d} \underline{t}}+\underline{J_{0}} \underline{C_{\mathrm{fsr}}} \\
& \times \operatorname{sign}\left(\frac{\mathrm{d} \theta_{\mathrm{e}}}{\mathrm{d} t}\right)=\underline{J_{0}} \underline{i_{\mathrm{b}}}
\end{aligned}
$$

avec :

$$
\frac{D_{\mathrm{r}}}{J_{\mathrm{r}} \omega_{\Phi}}=\left(\omega_{\Phi} T_{\mathrm{mr}}\right)^{-1}=\underline{T_{\mathrm{mr}}^{-1}} \rightarrow T_{\mathrm{mr}}=\frac{J_{\mathrm{r}}}{D_{\mathrm{r}}}
$$

constante de temps mécanique de la charge et de l'actionneur, ramenée au référentiel commun.

$$
\begin{gathered}
\frac{p}{J_{\mathrm{r}} \omega_{\Phi}^{2}}=\frac{1}{J_{\mathrm{r}}}=\frac{1}{J_{0}} \rightarrow \underline{J_{0}^{-1} C_{\mathrm{em} \max }^{\mathrm{ca}}} \rightarrow \underline{C_{\mathrm{fsr}}}=\frac{C_{\mathrm{fsr}}}{C_{\mathrm{em} \mathrm{max}}^{\mathrm{ca}}} \\
\frac{p K_{\Phi} \sqrt{2} U}{J_{\mathrm{r}} \omega_{\Phi}^{2} R}=\frac{J_{0}}{J_{\mathrm{r}}}=\underline{J_{0}} .
\end{gathered}
$$

En résumé, les équations adimensionnelles de la machine à aimant s'écrivent :

$$
\begin{aligned}
& \cos \eta=\underline{i_{\mathrm{a}}}+\underline{T_{\mathrm{e} 0}} \frac{\mathrm{d} i_{\mathrm{a}}}{\mathrm{d} \underline{t}}-\underline{T_{\mathrm{e} 0}} \underline{i_{\mathrm{b}}} \frac{\mathrm{d} \theta_{\mathrm{e}}}{\mathrm{d} \underline{t}} \\
& \sin \eta=\underline{i_{\mathrm{b}}}+\underline{T_{\mathrm{e} 0}} \frac{\mathrm{d} \underline{i_{\mathrm{b}}}}{\mathrm{d} \underline{t}}+\left(\underline{T_{\mathrm{e} 0}} \underline{i_{\mathrm{a}}}+\underline{T_{\mathrm{em} 0}^{-1}}\right) \frac{\mathrm{d} \theta_{\mathrm{e}}}{\mathrm{d} \underline{t}}
\end{aligned}
$$

$$
\frac{\mathrm{d}^{2} \theta_{\mathrm{e}}}{\mathrm{d} \underline{t}^{2}}+\underline{T_{\mathrm{mr}}^{\prime}} \frac{\mathrm{d} \theta_{\mathrm{e}}}{\mathrm{d} \underline{t}}+\underline{J}_{0} \underline{C_{\mathrm{frr}}} \operatorname{sign}\left(\frac{\mathrm{d} \theta_{\mathrm{e}}}{\mathrm{d} t}\right)=\underline{J}_{0} \underline{i}_{\mathrm{b}} .
$$

3.2 Machine À Reluctance $\left(K_{\phi}=0\right)$. - En prenant ici le temps de base correspondant à la période des oscillations libres de la MRV [3] :

$$
\omega_{\phi \mathrm{r}}=\sqrt{\frac{4 K_{\mathrm{r}}^{2} I^{2}}{L_{\mathrm{p}} J_{0}}} .
$$

Les équations du mouvement électrique s'écrivent d'une façon similaire à (31), comme :

$$
\begin{aligned}
\cos \eta=\underline{i}_{\mathrm{a}} & +\omega_{\Phi_{\mathrm{r}}}\left(\frac{L_{0}}{R}+\frac{L_{\mathrm{p}}}{R}\right) \frac{\mathrm{d} \underline{i}_{\mathrm{a}}}{\mathrm{d} \underline{t}} \\
& -\omega_{\Phi_{\mathrm{r}}}\left(\frac{L_{0}}{R}-\frac{L_{\mathrm{p}}}{R}\right) \underline{i_{\mathrm{b}}} \frac{\mathrm{d} \theta_{\mathrm{e}}}{\mathrm{d} \underline{t}} \\
\sin \eta=\underline{i}_{\mathrm{b}}+\omega_{\Phi_{\mathrm{r}}}\left(\frac{L_{0}}{R}-\frac{L_{\mathrm{p}}}{R}\right) \frac{\mathrm{d} \underline{i}_{\mathrm{b}}}{\mathrm{d} \underline{t}} & +\omega_{\Phi_{\mathrm{r}}}\left(\frac{L_{0}}{R}+\frac{L_{\mathrm{p}}}{R}\right) \underline{i_{\mathrm{a}}} \frac{\mathrm{d} \theta_{\mathrm{e}}}{\mathrm{d} \underline{t}} .
\end{aligned}
$$

L'équation du mouvement mécanique se déduit de (26), où $K_{\phi}=0$, comme :

$$
\begin{aligned}
\frac{\mathrm{d}^{2} \theta_{\mathrm{e}}}{\mathrm{d} \underline{t}^{2}}+\frac{D_{\mathrm{r}}}{J_{\mathrm{r}}} \omega_{\Phi_{\mathrm{r}}}^{-1} \frac{\mathrm{d} \theta_{\mathrm{e}}}{\mathrm{d} \underline{t}}+\frac{\frac{N_{\mathrm{dr}}}{2}}{J_{\mathrm{r}} \omega_{\Phi_{\mathrm{r}}}^{2}} & C_{\mathrm{fsr}} \operatorname{sign}\left(\frac{\mathrm{d} \theta_{\mathrm{e}}}{\mathrm{d} t}\right) \\
& =\frac{N_{\mathrm{dr}}^{2} L_{\mathrm{p}} U^{2}}{J_{\mathrm{r}} \omega_{\Phi_{\mathrm{r}}}^{2} R^{2}} i_{\mathrm{a}} \underline{i_{\mathrm{b}}}
\end{aligned}
$$

de la même façon que l'équation (33), car :

$$
\frac{2 L_{\mathrm{p}} \frac{N_{\mathrm{dr}}}{2} i_{\mathrm{a}} i_{\mathrm{b}}}{J_{\mathrm{r}} \frac{\sqrt{2} U}{R} \frac{\sqrt{2} U}{R}} \cdot \frac{2 U^{2}}{R^{2}}=\frac{2 L_{\mathrm{p}} N_{\mathrm{dr}} U^{2}}{J_{\mathrm{r}} R^{2}} \underline{i_{\mathrm{a}}} \underline{i_{\mathrm{b}}}
$$

en multipliant cette dernière expression par $N_{\mathrm{dr}} / 2 / \omega_{\phi r}^{2}$, on obtient le second membre de (37):

$$
\begin{aligned}
\frac{N_{\mathrm{dr}}^{2} L_{\mathrm{p}} U^{2}}{J_{\mathrm{r}} \omega_{\Phi_{\mathrm{r}}}^{2} R^{2}} i_{\mathrm{a}} \underline{i_{\mathrm{b}}} & =\frac{N_{\mathrm{dr}}^{2} L_{\mathrm{p}} I^{2}}{\frac{J_{\mathrm{r}} 4\left(\frac{N_{\mathrm{dr}}}{2} L_{\mathrm{p}}\right)^{2}}{i_{\mathrm{a}}} \underline{i_{\mathrm{b}}}} \\
& =\underline{J_{0}} \underline{i_{\mathrm{a}}} \underline{i_{\mathrm{b}}} .
\end{aligned}
$$

De plus, avec :

$$
\frac{\frac{N_{\mathrm{dr}}}{2}}{J_{\mathrm{r}} \omega_{\Phi_{\mathrm{r}}}^{2}}=\frac{N_{\mathrm{dr}}}{2 J_{\mathrm{r}} \frac{4 K_{\mathrm{r}}^{2} I^{2}}{L_{\mathrm{p}} J_{0}}}=\frac{J_{0}}{C_{\mathrm{em} \mathrm{max}}^{\mathrm{cf}}}
$$

et :

$$
\left(\omega_{\phi \mathrm{r}} T_{\mathrm{mr}}^{\prime}\right)^{-1}=\underline{T}_{\mathrm{mr}}^{\prime-1} .
$$


L'équation (37) se met sous la forme adimensionnelle complète :

$$
\frac{\mathrm{d}^{2} \theta_{\mathrm{e}}}{\mathrm{d} \underline{t}^{2}}+\underline{T_{\mathrm{mr}}^{\prime-1}} \frac{\mathrm{d} \theta_{\mathrm{e}}}{\mathrm{d} \underline{t}}+\underline{J_{0}} \underline{C_{\mathrm{fsr}}} \operatorname{sign}\left(\frac{\mathrm{d} \theta_{\mathrm{e}}}{\mathrm{d} t}\right)=\underline{J_{0}} \underline{i_{\mathrm{a}}} \underline{i_{\mathrm{b}}} .
$$

En résumé, les expressions des mouvements électriques et mécaniques, pour une machine à réluctance, s'écrivent :

$\left.\begin{array}{l}\cos \eta=\underline{i_{\mathrm{a}}}+\left(\underline{T_{\mathrm{e} 0}^{\prime}}+\underline{T_{\mathrm{ep}}}\right) \frac{\mathrm{d} i_{\mathrm{a}}}{\mathrm{d} \underline{t}}-\left(\underline{T_{\mathrm{e} 0}^{\prime}}-\underline{T_{\mathrm{ep}}}\right) \underline{i_{\mathrm{b}}} \frac{\mathrm{d} \theta_{\mathrm{e}}}{\mathrm{d} \underline{t}} \\ \sin \eta=\underline{i_{\mathrm{b}}}+\left(\underline{T_{\mathrm{e} 0}^{\prime}}+\underline{T_{\mathrm{ep}}}\right) \frac{\mathrm{d} \underline{\mathrm{b}}_{\mathrm{b}}}{\mathrm{d} \underline{t}}+\left(\underline{T_{\mathrm{e} 0}^{\prime}}-\underline{T_{\mathrm{ep}}}\right) \underline{i_{\mathrm{a}}} \frac{\frac{\mathrm{d} \theta_{\mathrm{e}}}{\mathrm{d} \underline{t}}}{\mathrm{~d}^{2} \theta_{\mathrm{e}}}+\underline{T_{\mathrm{mr}}^{\prime-1}} \frac{\mathrm{d} \theta_{\mathrm{e}}}{\mathrm{d} t}+\underline{J_{0}} \underline{C_{\mathrm{fsr}}} \operatorname{sign}\left(\frac{\mathrm{d} \theta_{\mathrm{e}}}{\mathrm{d} t}\right)=\underline{J_{0}} \underline{i_{\mathrm{a}}} \underline{i_{\mathrm{b}}}\end{array}\right\}$

avec :

$$
\omega_{\Phi_{\mathrm{r}}} \frac{L_{0}}{R}=\underline{T_{\mathrm{e} 0}^{\prime}} \text { et } \omega_{\Phi_{\mathrm{r}}} \frac{L_{\mathrm{p}}}{R}=\underline{T_{\mathrm{ep}}} .
$$

On remarque aisément une forme quasi-identique des équations (35) et (38) : ce qui permet, par la suite, l'établissement d'un programme de calcul unique pour l'exploration de toutes les machines pas à pas.

Par ailleurs, les systèmes (35) et (38) sont très similaires aux expressions adimensionnelles des machines synchrones [4], où l'excitation est matérialisée par une composante homopolaire du courant.

\section{Considérations générales sur la modélisation du} fonctionnement dynamique.

En assimilant la dynamique des machines pas à pas à celle des machines synchrones, alimentées par les impulsions de tension (courant), nous avons pu établir les trois ensembles d'équations, que nous regroupons sous une forme directement programmable :

a) EQUATIONS DANS UN REPÈRE IMMOBILE $(\alpha, \beta)$ DU STATOR.

- pour MP (ou MH)

$$
\left.\begin{array}{rl}
u_{\alpha}= & R i_{\alpha}+L_{0} \frac{\mathrm{d} i_{\alpha}}{\mathrm{d} t}-K_{\Phi} \Omega \sin p \theta_{\mathrm{m}} \\
u_{\beta}= & R i_{\beta}+L_{0} \frac{\mathrm{d} i_{\beta}}{\mathrm{d} t}+K_{\Phi} \Omega \cos p \theta_{\mathrm{m}} \\
C_{\mathrm{em}}= & -K_{\Phi}\left(i_{\alpha} \sin p \theta_{\mathrm{m}}-i_{\beta} \cos p \theta_{\mathrm{m}}\right)=C_{\mathrm{frr}}(\operatorname{sign} \Omega) \\
& -D_{\mathrm{r}} \Omega-J_{\mathrm{r}} \frac{\mathrm{d} \Omega}{\mathrm{d} t}
\end{array}\right\}
$$

- pour MRV:

$u_{\alpha}=R i_{\alpha}+\left(L_{0}+L_{\mathrm{p}} \cos N_{\mathrm{dr}} \theta_{\mathrm{m}}\right) \frac{\mathrm{d} i_{\alpha}}{\mathrm{d} t}+L_{\mathrm{p}} \sin N_{\mathrm{dr}} \theta_{\mathrm{m}} \frac{\mathrm{d} i_{\beta}}{\mathrm{d} t}-\left(K_{\mathrm{r}} i_{\alpha} \sin N_{\mathrm{dr}} \theta_{\mathrm{m}}-K_{\mathrm{r}} i_{\beta} \cos N_{\mathrm{dr}} \theta_{\mathrm{m}}\right) \Omega$

$u_{\beta}=R i_{\beta}+L_{\mathrm{p}} \sin N_{\mathrm{dr}} \theta_{\mathrm{m}} \frac{\mathrm{d} i_{\alpha}}{\mathrm{d} t}+\left(L_{0}-L_{\mathrm{p}} \cos N_{\mathrm{dr}} \theta_{\mathrm{m}}\right) \frac{\mathrm{d} i_{\beta}}{\mathrm{d} t}+2\left(K_{\mathrm{r}} i_{\alpha} \cos N_{\mathrm{dr}} \theta_{\mathrm{m}}+K_{\mathrm{r}} i_{\beta} \sin N_{\mathrm{dr}} \theta_{\mathrm{m}}\right) \Omega$

$C_{\mathrm{em}}=-K_{\mathrm{r}}\left[\left(i_{\alpha}^{2}-i_{\beta}^{2}\right) \sin N_{\mathrm{dr}} \theta_{\mathrm{m}}-2 i_{\alpha} i_{\beta} \cos N_{\mathrm{dr}} \theta_{\mathrm{m}}\right]=C_{\mathrm{fsr}} \operatorname{sign}(\Omega)+D_{\mathrm{r}} \Omega+J_{\mathrm{r}} \frac{\mathrm{d} \Omega}{\mathrm{d} t}$.

b) EQUATIONS DANS UN REPÈRE MOBILE $(a, b)$ DU ROTOR.

$$
\begin{gathered}
- \text { pour MP (ou MH) : } \\
\frac{\mathrm{d} i_{\mathrm{a}}}{\mathrm{d} t}=-\frac{R}{L_{0}} i_{\mathrm{a}}+p i_{\mathrm{b}} \Omega+\frac{1}{L_{0}} U_{\mathrm{a}} \\
\frac{\mathrm{d} i_{\mathrm{b}}}{\mathrm{d} t}=-p i_{\mathrm{a}} \Omega-\frac{R}{L_{0}} i_{\mathrm{b}}-\frac{K_{\phi}}{K_{0}} \Omega+\frac{1}{L_{0}} U_{\mathrm{b}} \\
\frac{\mathrm{d} \Omega}{\mathrm{d} t}=\frac{K_{\phi}}{J_{\mathrm{r}}} i_{\mathrm{b}}-\frac{D_{\mathrm{r}}}{J_{\mathrm{r}}} \Omega-\frac{C_{\mathrm{fsr}}}{J_{\mathrm{r}}} \operatorname{sign}(\Omega)
\end{gathered}
$$

\section{- pour MRV:}

$$
\begin{aligned}
& \frac{\mathrm{d} i_{\mathrm{a}}}{\mathrm{d} t}=-\frac{R}{L_{0}+L_{\mathrm{p}}} i_{\mathrm{a}}+p \frac{L_{0}-L_{\mathrm{p}}}{L_{0}+L_{\mathrm{p}}} \Omega i_{\mathrm{b}}+\frac{1}{L_{0}+L_{\mathrm{p}}} U_{\mathrm{a}} \\
& \frac{\mathrm{d} i_{\mathrm{b}}}{\mathrm{d} t}=-p \frac{\left(L_{0}+L_{\mathrm{p}}\right)}{L_{0}-L_{\mathrm{p}}} \Omega i_{\mathrm{a}} \\
& -\frac{R}{L_{0}-L_{\mathrm{p}}} i_{\mathrm{b}}+\frac{1}{L_{0}-L_{\mathrm{p}}} U_{\mathrm{b}}
\end{aligned}
$$

$$
\frac{\mathrm{d} \Omega}{\mathrm{d} t}=\frac{2 K_{\mathrm{r}}}{J_{\mathrm{r}}} i_{\mathrm{a}} i_{\mathrm{b}}-\frac{D_{\mathrm{r}}}{J_{\mathrm{r}}} \Omega-\frac{C_{\mathrm{fsr}}}{J_{\mathrm{r}}} \operatorname{sign}(\Omega)
$$


c) EQUATIONS ADIMENSIONNELLES DANS UN REPÈRE MOBILE (a, b) DU ROTOR.

- pour MP (ou MH):

$$
\begin{aligned}
& \frac{\mathrm{d} i_{\mathrm{a}}}{\mathrm{d} \underline{t}}=-\frac{1}{\underline{T_{\mathrm{e} 0}}} \underline{i}_{\mathrm{a}}+\frac{\mathrm{d} \theta_{\mathrm{e}}}{\mathrm{d} \underline{t}} \underline{i_{\mathrm{b}}}+\frac{1}{\underline{T_{\mathrm{e} 0}}} \cos \eta \\
& \frac{\mathrm{d} i_{\mathrm{b}}}{\mathrm{d} \underline{t}}=-\underline{i_{\mathrm{a}}} \cdot \frac{\mathrm{d} \theta_{\mathrm{e}}}{\mathrm{d} \underline{t}}-\frac{1}{T_{\mathrm{e} 0}} \underline{i_{\mathrm{b}}}- \\
& -\frac{1}{\underline{T_{\mathrm{e} 0}} \underline{T_{\mathrm{em} 0}}} \frac{\mathrm{d} \theta_{\mathrm{e}}}{\mathrm{d} t}+\frac{1}{\underline{T_{\mathrm{e} 0}}} \sin \eta \\
& \frac{\mathrm{d}^{2} \theta_{\mathrm{e}}}{\mathrm{d} \underline{t}^{2}}=\underline{J_{0}} \underline{i}_{\mathrm{b}}-\underline{T_{\mathrm{mr}}^{-1}} \frac{\mathrm{d} \theta_{\mathrm{e}}}{\mathrm{d} \underline{t}}-\underline{J_{0}} \underline{C_{\mathrm{fsr}}} \operatorname{sign}\left(\frac{\mathrm{d} \theta_{\mathrm{e}}}{\mathrm{d} \underline{t}}\right) \\
& \text { _ pour MRV : } \\
& \frac{\mathrm{d} i_{\mathrm{a}}}{\mathrm{d} \underline{t}}=-\frac{1}{\underline{T_{\mathrm{e} 0}^{\prime}}+\underline{T_{\mathrm{ep}}}} i_{\mathrm{a}}+\frac{T_{\mathrm{e} 0}^{\prime}-\frac{T_{\mathrm{ep}}}{\bar{T}_{e 0}^{\prime}}+\underline{T_{\mathrm{ep}}}}{} \times \\
& \times \underline{i_{\mathrm{b}}} \frac{\mathrm{d} \theta_{\mathrm{e}}}{\mathrm{d} t}-\frac{1}{\underline{T_{\mathrm{e} 0}^{\prime}}+\underline{T_{\mathrm{ep}}}} \cos \eta \\
& \frac{\mathrm{d} i_{\mathrm{b}}}{\mathrm{d} \underline{t}}=-\frac{T_{\mathrm{e} 0}^{\prime}}{\overline{T_{\mathrm{e} 0}^{\prime}}}-\underline{T_{\mathrm{ep}}}{\underline{T_{\mathrm{ep}}}}_{\underline{\mathrm{a}}} \cdot \frac{\mathrm{d} \theta_{\mathrm{e}}}{\mathrm{d} \underline{t}}- \\
& -\frac{1}{\underline{T_{\mathrm{e} 0}^{\prime}}-\underline{T_{\mathrm{ep}}}} i_{\mathrm{b}}+\frac{1}{\underline{T_{\mathrm{e} 0}^{\prime}}-\underline{T_{\mathrm{ep}}}} \sin \eta
\end{aligned}
$$

Dans toutes ces expressions, la charge résistante $\left(C_{\mathrm{r}}\right)$ est considérée comme une combinaison d'inertie de frottement sec et de frottement visqueux et les paramètres correspondants sont cumulés avec ceux de l'actionneur $\left(C_{\mathrm{fs} 0}, D_{0}, J_{\mathrm{r} 0}\right)$.

Classiquement, le passage des coordonnées immobiles (naturelles) vers les coordonnées mobiles, puis vers la notation adimensionnelle, a pour but de simplifier l'écriture des équations et permet les résolutions numériques les plus simples possible. Par ailleurs, l'écriture de forme (43), (44), unifie les deux types de machines régies par des principes différents et conduit plus aisément à des conclusions générales.

Le désavantage de l'écriture adimensionnelle apparaît surtout lorsque l'on doit établir la liaison entre l'alimentation électroni ue et la machine «normalisée ". C'est pourquoi, certains chercheurs préfèrent manier l'écriture dans les coordonnées immobiles, qui, quoique plus lourde, présente l'avantage de s'intégrer immédiatement dans les équations des circuits électroniques pilotant l'actionneur. Un tel raisonnement est d'autant plus fondé que les micro-ordinateurs modernes mettent à la portée de tous une capacité de calcul suffisamment importante, pour prendre en compte les transformations analytiques (comme le passage matriciel (17), (18), (19) et (20)), et n'ont pas besoin de formulations allégées.
En ce qui nous concerne, tout en présentant l'ensemble des écritures possibles, nous préférons toujours la formulation dans les coordonnées mobiles sous une forme adimensionnelle et ceci pour deux raisons :

— pédagogiques d'abord: la généralisation de l'écriture bien assimilée conduit toujours à une meilleure compréhension du phénomène étudié ;

- numériques par la suite : la résolution des équations par la méthode Runge-Kutta permet un algorithme interactif identique pour l'ensemble des actionneurs et assure une convergence rapide dans tous les cas de figure que nous avons pu tester; par ailleurs, les fonctions graphiques adimensionnelles sont plus faciles à analyser.

\section{Remarque.}

Lorsque les constantes de temps électriques peuvent être négligées (alimentation en courant, inertie mécanique très importante ...), l'élimination de $\underline{T}_{\mathrm{e} 0}$ dans (43) conduit, pour une machine à aimant, à :

$$
\begin{gathered}
\sin \eta=\underline{i_{\mathrm{b}}}+\underline{T_{\mathrm{em} 0}^{-1}} \frac{\mathrm{d} \theta_{\mathrm{e}}}{\mathrm{d} \underline{t}} \\
\frac{\mathrm{d}^{2} \theta_{\mathrm{e}}}{\mathrm{d} \underline{t}^{2}}+\underline{T_{\mathrm{mr}}^{-1}} \frac{\mathrm{d} \theta_{\mathrm{e}}}{\mathrm{d} \underline{t}}+\underline{J_{0}} \underline{C_{\mathrm{fsr}}} \operatorname{sign}\left(\frac{\mathrm{d} \theta_{\mathrm{e}}}{\mathrm{d} t}\right)=\underline{J_{0}} \underline{i_{\mathrm{b}}}
\end{gathered}
$$

qui, à son tour, se réduit à l'expression :

$$
\begin{aligned}
\frac{\mathrm{d}^{2} \theta_{\mathrm{e}}}{\mathrm{d} \underline{t}^{2}}+\left(\underline{T_{\mathrm{mr}}^{-1}-J_{0}} \underline{T}_{\mathrm{em} 0}^{-1}\right) \frac{\mathrm{d} \theta_{\mathrm{e}}}{\mathrm{d} \underline{t}} & \\
& +\underline{J_{0}} \underline{C_{\mathrm{fsr}}} \operatorname{sign}\left(\frac{\mathrm{d} \theta_{\mathrm{e}}}{\mathrm{d} t}\right)=\underline{J}_{0} \sin \eta
\end{aligned}
$$

d'un élément oscillatoire du second ordre, identique en principe à celui des formules linéarisées [3]. Il est intéressant de constater que la même hypothèse, concernant une MRV (soit $\underline{T}_{\mathrm{e} 0}^{\prime}=0$ dans (44)), conduit à des systèmes oscillatoires d'ordre supérieur.

\section{Fonctionnement dynamique stable.}

5.1 MACHINE DE RÉFÉRENCE. — L'analyse du mounumérique des équations (43), (44).

Pour les études numériques, nous avons choisi une machine hybride de faible résolution $\left(N_{\mathrm{pt}}=40\right)$, alimentée en tension ou en courant par une ou plusieurs voies. Les paramètres correspondants sont affichés sur chaque série de courbes.

5.2 POSITIONNEMENT APRÈS UNE IMPULSION. - La figure 8 compare les oscillations du rotor sans charge, pour une alimentation en tension: "une phase à la fois » (Fig. 8a) et "deux phases à la fois » (Fig. 8b). 
Le temps d'annulation des vibrations, $t_{\mathrm{nv}}$, est de l'ordre de $20 \mathrm{~ms}$, pour une machine alimentée "deux phases à la fois", et beaucoup plus long $(\sim 65 \mathrm{~ms})$, pour une machine alimentée par une seule phase. Ceci s'explique par l'allure des courants transitoires $i_{\alpha}$ et $i_{\beta}$, qui, dirigés pratiquement dans le sens contraire, dans les deux phases alimentées, se ferment à l'intérieur de la machine, et par la dissipation d'énergie correspondante, qui constitue un amortisseur naturel des vibrations. Le phénomène est encore plus flagrant dans une machine, dont une phase est alimentée en tension et l'autre court-circuitée (Fig. 8c). Ici, l'évolution transitoire du courant induit dans la phase court-circuitée, $i_{\alpha c c}$, permet la circulation de $i_{\beta}$ à l'intérieur de la machine et un " autofreinage " presque aussi efficace que sur la figure $8 \mathrm{~b}$. Par contre, l'alimentation en courant des deux phases à la fois, qui supprime totalement les régimes transitoires de $i_{\alpha}$ et $i_{\beta}$ (Fig. 8d), empêche le positionnement stable de la machine non chargée. Le couple résistant constant (Fig. 8e) et encore plus l'amortisseur visqueux (Fig. 8f) contribuent à la stabilisation dynamique sur un pas.
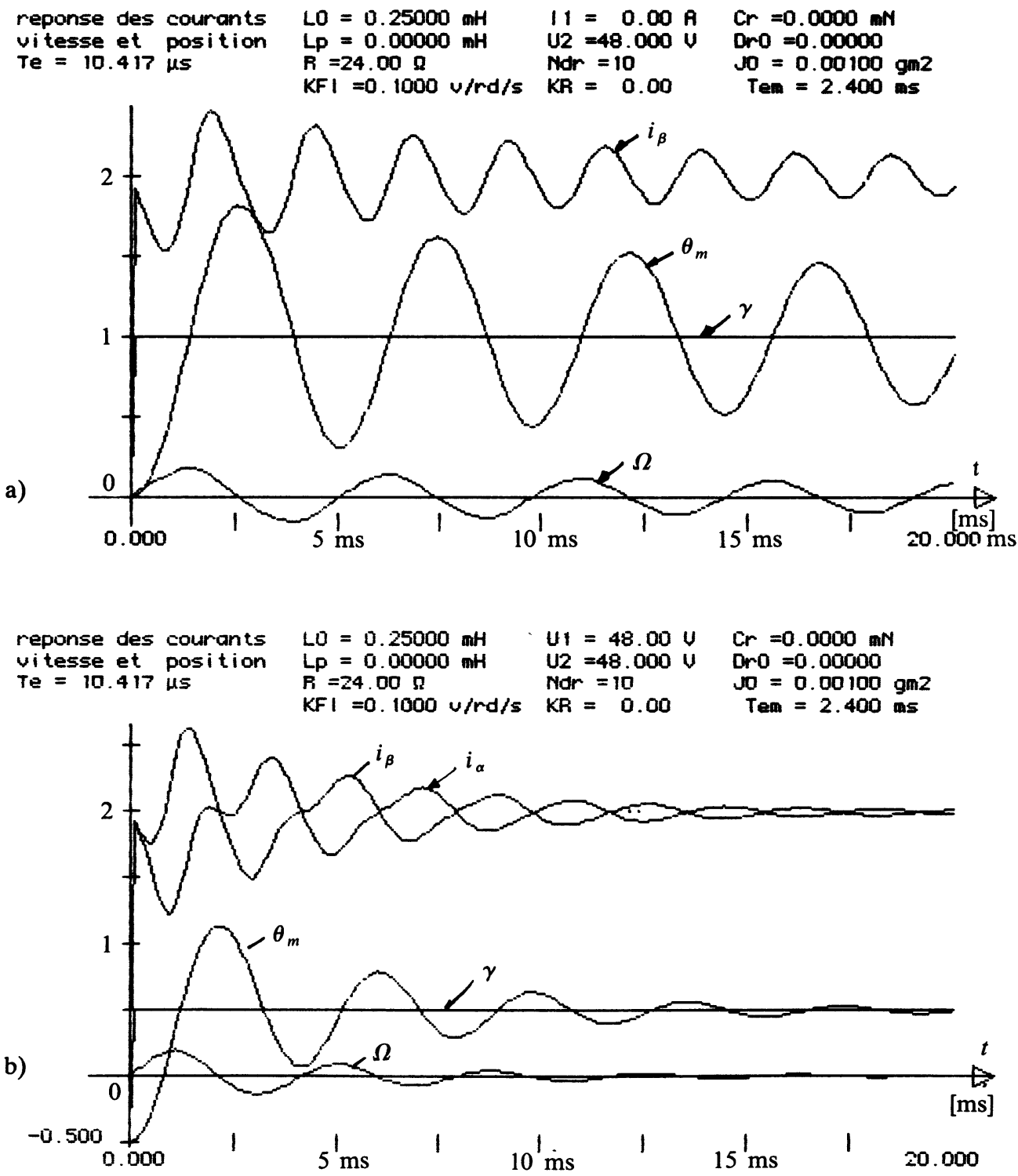

Fig. 8. - (a) Alimentation en tension d'une phase, l'autre étant en circuit ouvert. (b) Alimentation en tension des deux phases simultanément. (c) Alimentation en tension d'une phase, l'autre étant en circuit ouvert. (d) Alimentation en courant des deux phases simultanément. (e) Influence d'un couple résistant. (f) Influence d'un frottement visqueux.

[(a) Tension feeding for one phase on - open circuit for the other. (b) Tension feeding two phases on. (c) Tension feeding for one phase on - short circuit for the other. (d) Current feeding for both phases. (e) Influence of an applied torque. (f) Influence of a viscous friction.] 
reponse des courunts vitesse et position $T e=10.417 \mu s$

$L O=0.25000 \mathrm{mH}$ LP $=0.00000 \mathrm{mH}$ $R=24$ on $\Omega$

$u t=0.00 \mathrm{u}$ $12=48.0004$ $\mathrm{Mdr}=10$

$C r^{\circ}=0.0000 \mathrm{mr}$

$\mathrm{KFI}=0.1000 \mathrm{u} / \mathrm{rd} / \mathrm{s} \quad \mathrm{KR}=0.00$

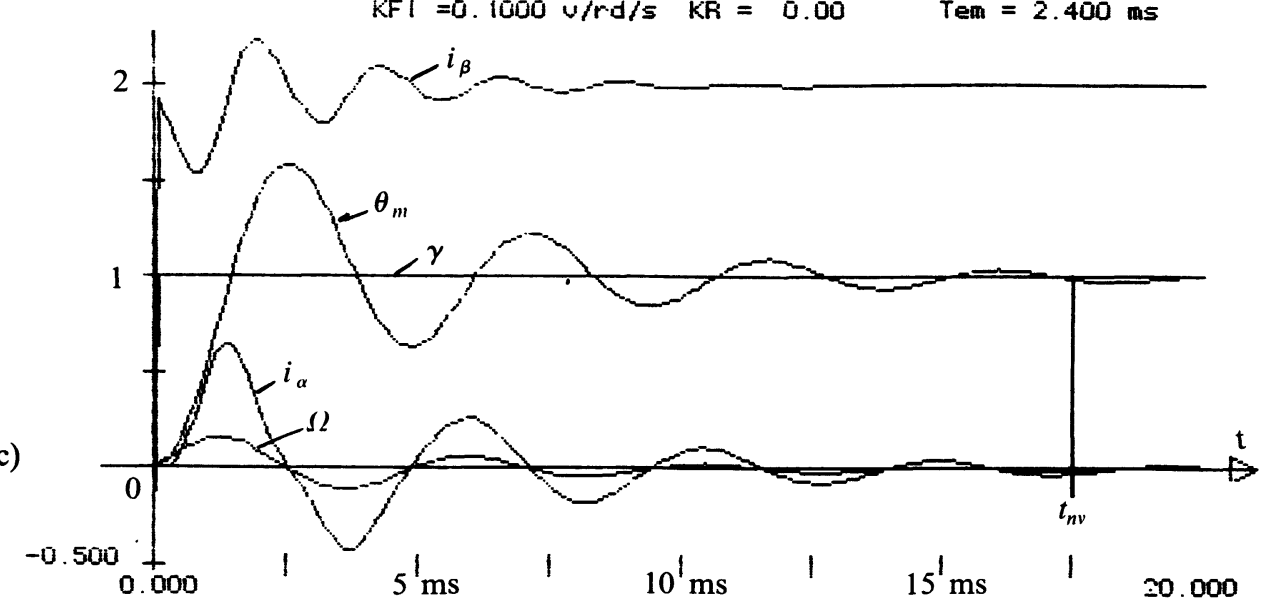

d)

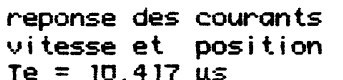
vitesse et position $\mathrm{Te}=10.417 \mu \mathrm{s}$

$L O=0.25000 \mathrm{mH}$ $L_{p}=0.00000 \mathrm{mH}$ $\begin{array}{lll}R=24.00 \Omega & N d r=10 & \text { Jo }=0.00100 \mathrm{gmz} \\ K F I=0.1000 \mathrm{u} / \mathrm{rd} / \mathrm{s} & \mathrm{KR}=0.00 & \text { Tem }=2.400 \mathrm{~ms}\end{array}$

$11=2.00 \mathrm{U} \quad \mathrm{Cr}=0.0000 \mathrm{mM}$

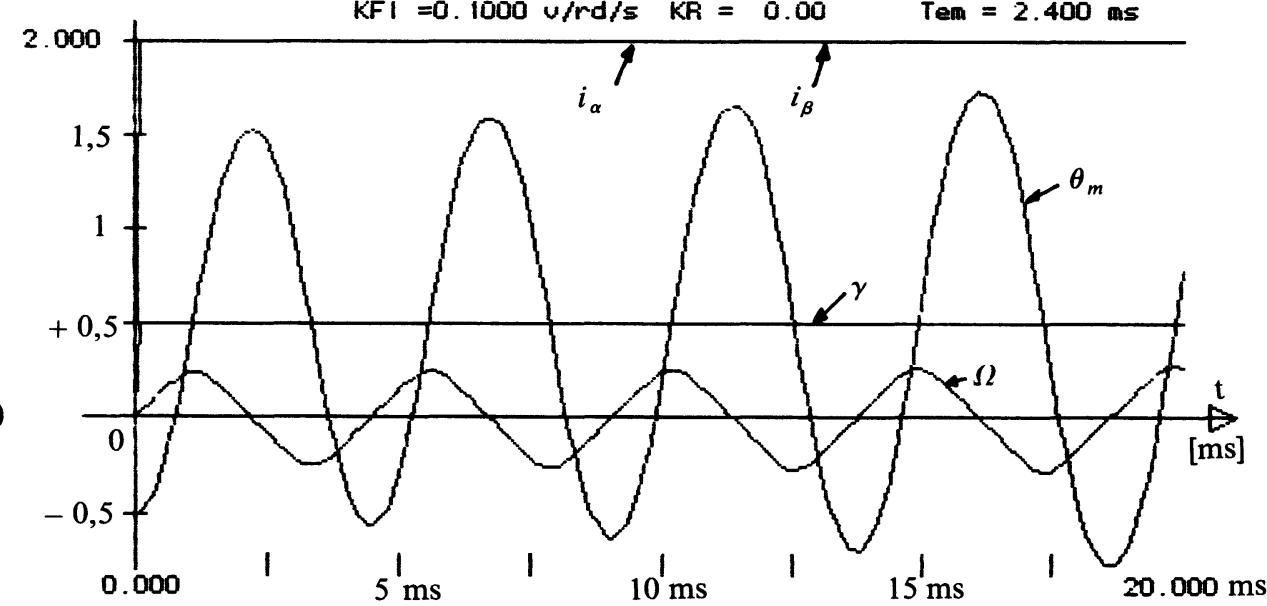

reponse des courants vitesse et position $\mathrm{Te}=10.417 \mathrm{\mu s}$

$\angle O=0.25000 \mathrm{mH}$ LP $=0.00000 \mathrm{mH}$ $R=24$. $00 \Omega$

$41=0.00 \mathrm{u}$ $12=48.000 \mathrm{U}$ Ndr $=10$

Oro $=0.00000$ $\mathrm{s} 0=0.00100 \mathrm{gm} 2$

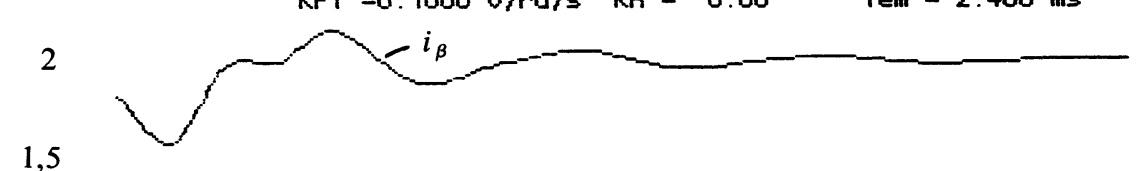

$\gamma$

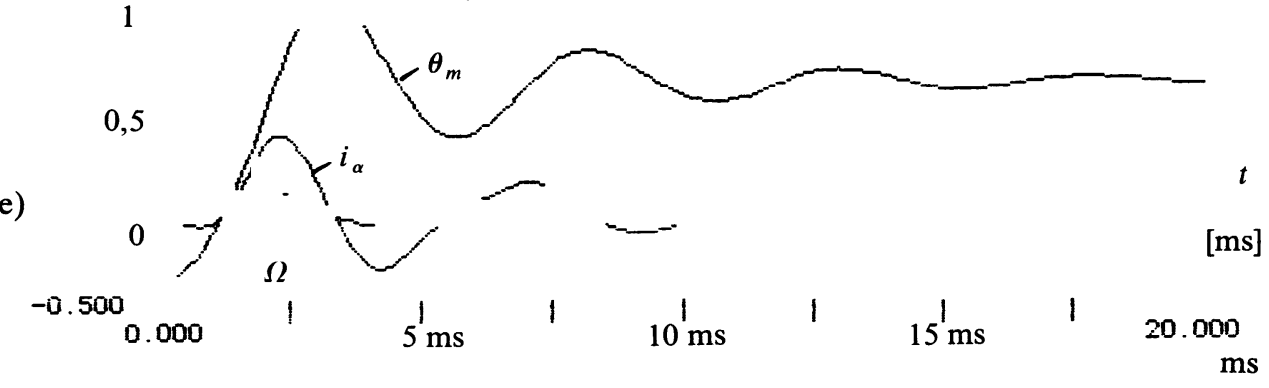

Fig. 8 (suite). 


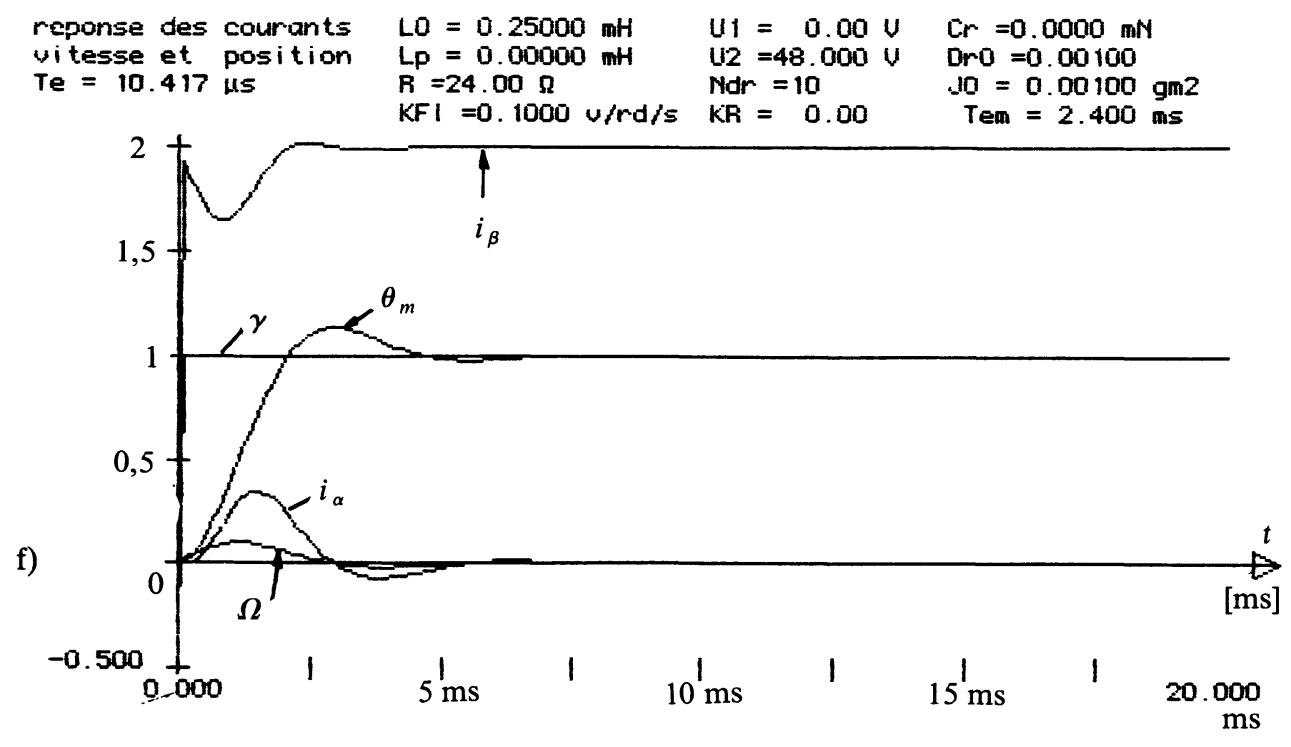

Fig. 8 (suite).

5.3 MOUVEMENT QUASI-DYNAMIQUE. - Le mouvement quasi-dynamique correspond à l'accomplissement par le rotor d'une série de pas de fréquence suffisamment faible, pour que le processus d'oscillation soit pratiquement amorti au commencement de chaque nouveau pas.

La fréquence maximale de fonctionnement quasidynamique est donc limitée par:

$$
f_{\mathrm{qd}}=\frac{1}{T_{\mathrm{qd}}} \leqslant \frac{1}{T_{\mathrm{nv}}}
$$

et la vitesse au début de chaque pas : $\theta_{\mathrm{m}}=0$. La figure 9a montre la simulation numérique du mouvement, où la machine effectue 5 pas, de période égale à $10 \mathrm{~ms}$, selon le cyclogramme $(1,2)(-1,2)(-1$, -2) $(1,-2)$, soit à une fréquence mécanique $f_{\mathrm{m}}=100 \mathrm{pas} / \mathrm{sec}$. Décomposons le fonctionnement quasi-dynamique à vide et en charge, en procédant par les étapes successives :

$A$ vide (Fig. 9b).

- Avant $t=0$, le rotor se trouve à l'équilibre stable, au point $\theta_{\mathrm{e}}=0\left(\theta_{\mathrm{m}}=0\right)$, correspondant au branchement d'une séquence de tension: $(1,-2)$ par exemple.

- A l'instant $t=0$, la tension est commutée de $(1,-2)$ vers $(1,2)$ et, pendant l'établissement du courant $i_{\alpha}=i_{\beta}=I$, le rotor effectue une trajectoire qui l'amène à $t=t_{1}$ au point d'équilibre $\mathrm{P}_{1}$ $\left(\theta_{\mathrm{e} 1}=p \theta_{\mathrm{m} 1}\right)$.

- A cause de la vitesse $\dot{\theta}_{\mathrm{m} \max }$ (Fig. 9c) et de l'énergie acquise (qui, à cet instant, sont maximales), le rotor continue son déplacement (contre le couple $C_{\mathrm{em}}$ ), pour atteindre une surrégulation $\Delta \theta_{\mathrm{m}}$; le mouvement oscillatoire, qui en résulte, est pratiquement amorti, lorsque l'énergie cinétique du rotor se transforme en pertes électriques (enroulement, fer) et mécaniques, donc en chaleur.

- A l'instant $t=t_{2} \geq t_{\mathrm{nv}}$, la commutation suivante fait répéter le processus décrit préalablement.

Si avant $t=0$, le rotor est chargé (3) (Fig. 10) :

- Son point de fonctionnement initial, $\mathrm{P}_{0}$, est ramené à l'arrière d'un angle :

$$
\theta_{\mathrm{e} 0}=\gamma+\eta=p \theta_{\mathrm{m} 0}
$$

par un couple résistant $C_{\mathrm{r}}$.

- Lorsqu'à l'instant $t=0$, la tension est commutée de $(1,-2)$ vers $(1,2)$, le mouvement du rotor se déroule de la même façon que pour le fonctionnement à vide, mais cette fois la position stable $\mathrm{P}_{1}\left(\theta_{\mathrm{e} 1}\right)$ est décalée en arrière par rapport à l'axe magnétique du champ (imposé par $\gamma(t)$ ) d'un angle du couple $\eta$ (Fig. 11). Cet angle est également visible à la première commutation de la figure $9 \mathrm{a}$.

Le mouvement quasi-dynamique est caractérisé par :

- la fréquence maximale des pas: pour les machines dotées d'amortisseurs efficaces et ayant, de ce fait, une réponse indicielle apériodique, on peut admettre :

$$
f_{\mathrm{qd} \max } \simeq \frac{1}{5} T_{\mathrm{ma}}
$$

avec $T_{\mathrm{ma}}$ : relaxation du mouvement apériodique ;

- la surrégulation $\Delta \theta_{\mathrm{m}}$, c'est-à-dire l'écart maximal transitoire du point d'équilibre pendant l'accomplissement d'un pas ;

( ${ }^{3}$ La machine préalablement alimentée ou possédant un couple de détente. 


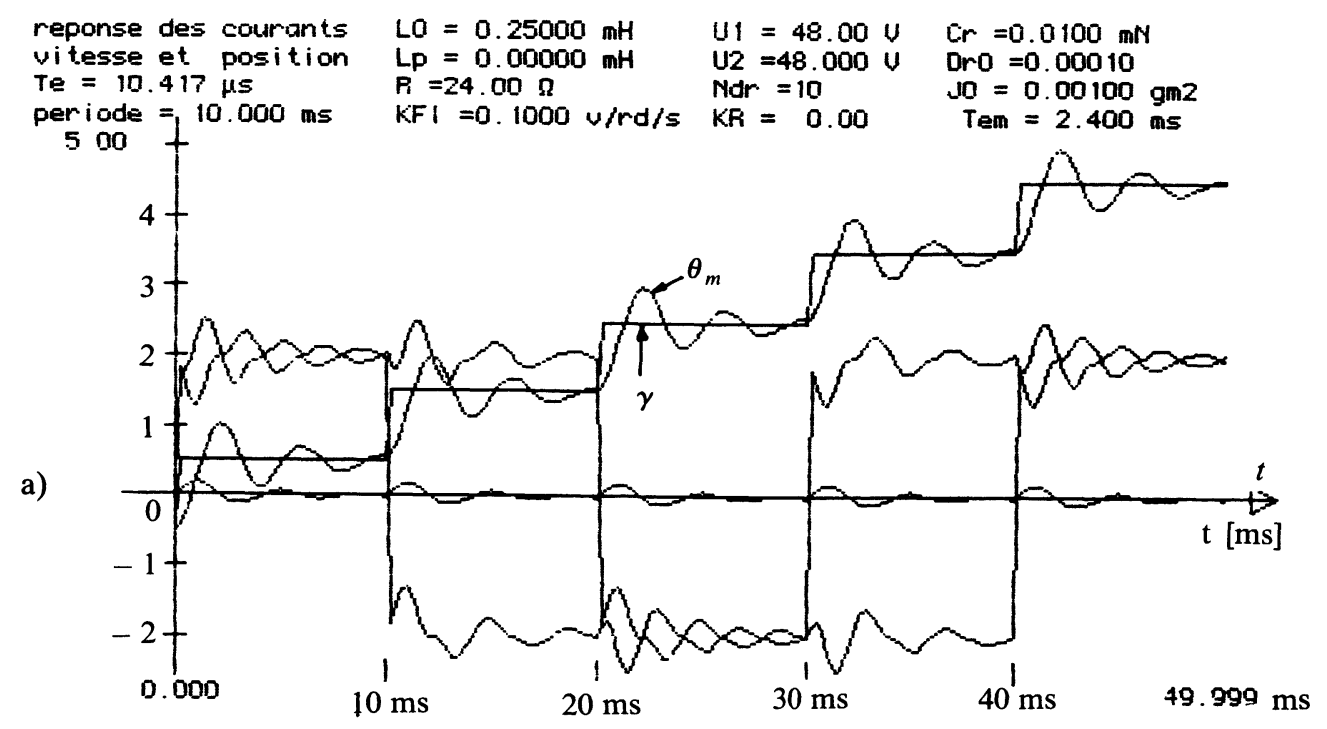

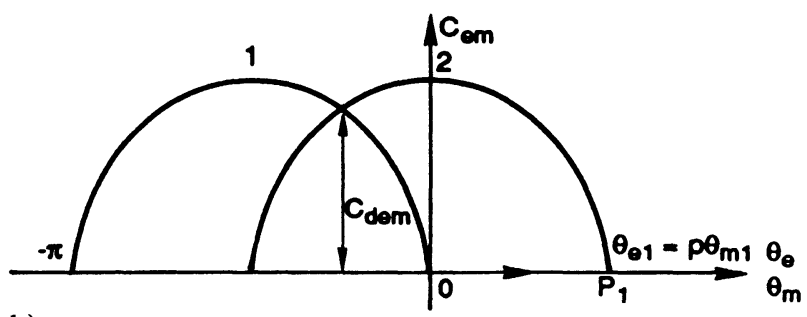

b)

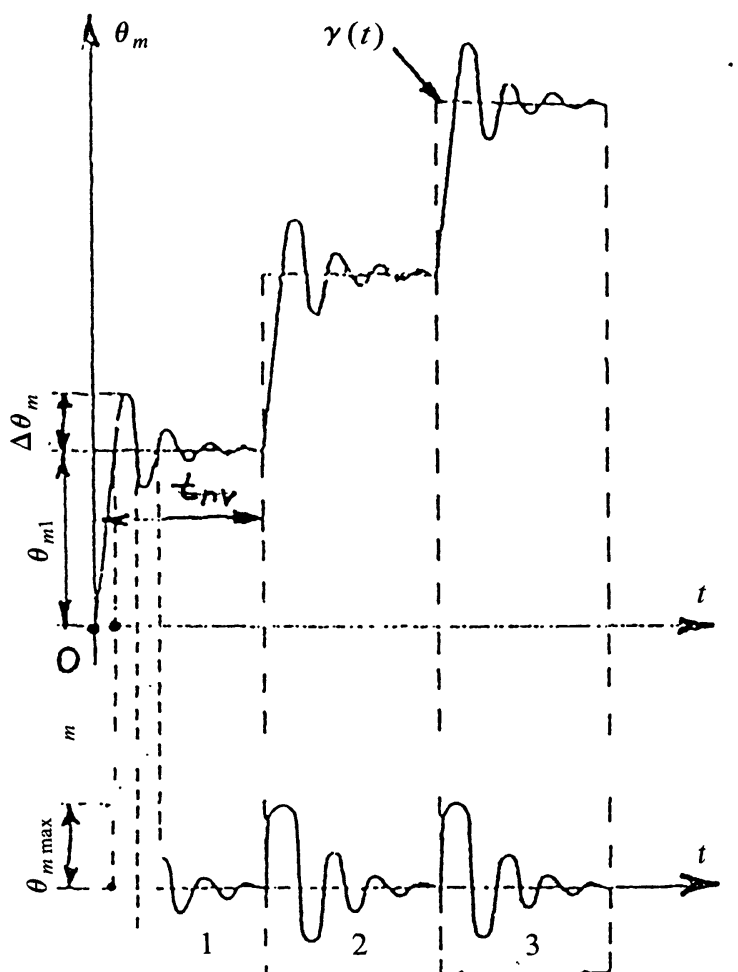

c)

Fig. 9. - (a) Franchissement de plusieurs pas. (b) Variation du couple avec la position. (c) Comportement dynamique idéalisé.

[(a) Step motor displacement. (b) Statical torque versus position. (c) Idealization of dynamic behaviour.]

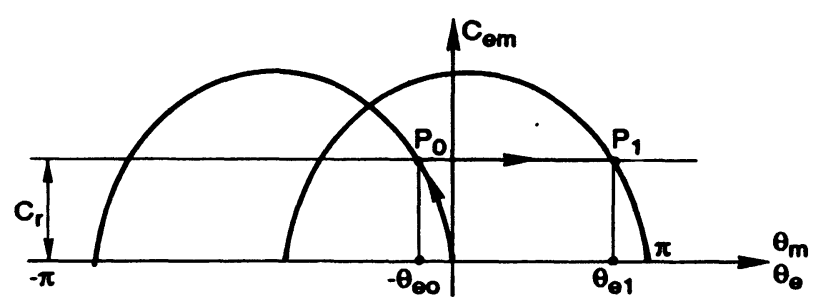

Fig. 10. - Variation du couple avec la position en présence d'un couple résistant.

[Torque versus position in the presence of an applied torque.]

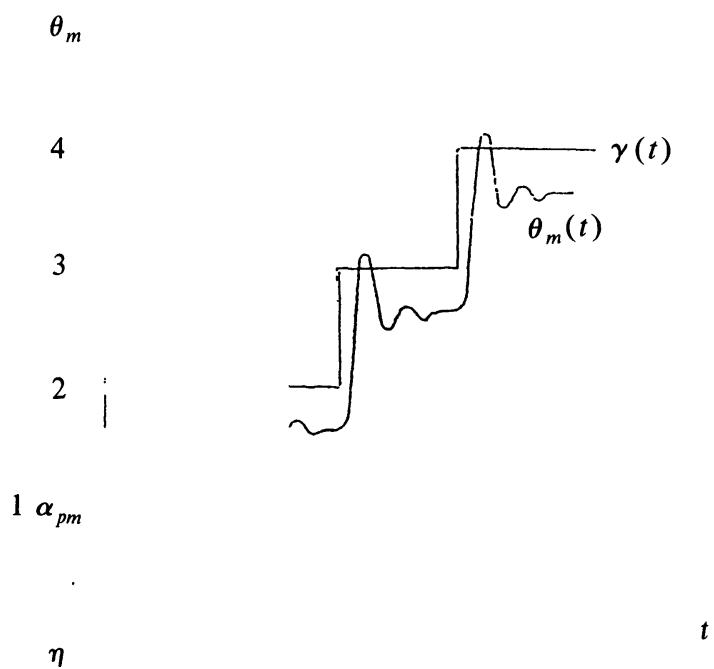

Fig. 11. - Comportement dynamique idéalisé en présence d'un couple résistant.

[Idealization of dynamical behaviour in the presence of an applied torque.] 
- la valeur maximale de la vitesse angulaire transitoire $\stackrel{\circ}{\boldsymbol{m} \max }_{\text {; }}$

- le temps d'annulation (amortissement) des vibrations $t_{\mathrm{nv}}$.

- Les dispositifs, dont la vocation première est le positionnement (après un ou plusieurs pas) et qui fonctionnent donc le plus souvent en mode quasidynamique, sont construits pour minimiser $t_{\mathrm{nv}}$ et $\Delta \theta_{\mathrm{m}}$ (par un système d'amortisseurs), tout en gardant une vitesse angulaire instantanée $\stackrel{\circ}{\mathrm{m}}_{\mathrm{m}}$ élevée.

5.4 MOUVEMENT DYNAMiQue ÉTABLI. - Le mouvement dynamique établi correspond à l'alimentation de la machine par des fréquences quelconques, de manière à opérer :

- un démarrage,

- un positionnement,
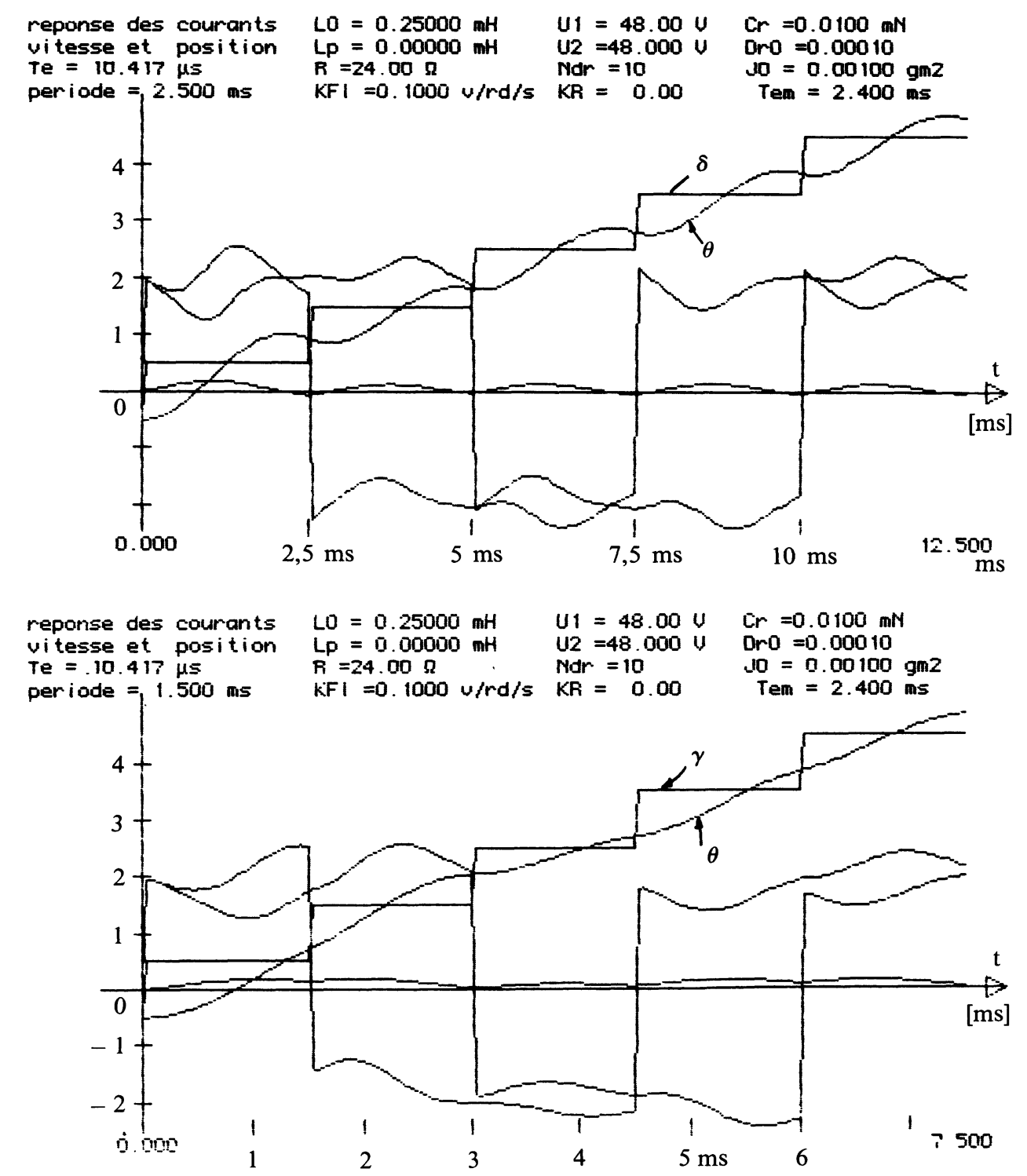

Fig. 12. - (a) Comportement dynamique à fréquence élevée. (b) Comportement dynamique quasi-synchrone.
- un fonctionnement à vitesse variable,

- un freinage (ou freinage - arrêt sur un pas),

- un changement de sens de rotation.

Plus la fréquence des commutations augmente (et donc plus leur période diminue), plus les oscillations de la position du rotor, $\theta_{m}(t)$, diminuent $\left({ }^{4}\right)$, comme en témoignent les simulations numériques des figures 12a (période de commutation : $T_{\mathrm{c}}=2,5 \mathrm{~ms}$ ) et $12 \mathrm{~b}$ (période de commutation : $T_{\mathrm{c}}=1,5 \mathrm{~ms}$ ). Pour une période de l'ordre de $1 \mathrm{~ms}$, la machine de référence, chargée avec un couple de $0,01 \mathrm{Nm}$, présente un fonctionnement pratiquement synchrone, soit :

$$
\gamma-\omega_{\mathrm{e}} t \cong \gamma-\omega t
$$

$\left({ }^{4}\right)$ Phénomènes de résonance exclus.

[(a) Dynamical behaviour at high frequency. (b) Almost synchronous behaviour.] 
Dans ces conditions, les calculs numériques pas par pas n'ont plus d'intérêt et il convient de rechercher, à partir des équations du mouvement, un certain nombre de caractéristiques globales, en fonction de l'utilisation de la machine étudiée.

La caractéristique mécanique du mouvement, soit la courbe couple-vitesse (ou couple-fréquence de commutation), se déduit numériquement à partir des expressions (43), (44), où l'on doit préciser :

- l'alimentation,

- les conditions initiales,

- la caractéristique de la charge.

Pour les tests numériques de la machine de référence, nous prenons donc:

- une alimentation "deux phases à la fois ", selon le cyclogramme de la figure 9 : ce qui permet de considérer les ondes fondamentales de la tension de la forme :

$$
\begin{aligned}
& U \cos \omega_{\mathrm{e}} t \\
& U \cos \left(\omega_{\mathrm{e}} t-\Pi / 2\right)
\end{aligned}
$$

- deux conditions initiales : l'une concernant le démarrage à partir de la charge statique, soit $\eta \neq 0, \theta_{\mathrm{m}} \neq 0, \theta_{\mathrm{m}}=0$, l'autre concernant l'accélération à partir d'une vitesse constante $\dot{\theta}_{\mathrm{m}}=\dot{\theta}_{\mathrm{m} 0}$;

- la charge extérieure est du type frottement sec (donc indépendante de la vitesse), couplée à une inertie indépendante de la position du rotor.

Le calcul commence à une fréquence mécanique : $f_{\mathrm{m}}=100 \mathrm{pas} / \mathrm{s}$ ( $n=150$ tours $\left./ \mathrm{min}\right)$, pour se terminer à : $f_{\mathrm{m}}=4000 \mathrm{pas} / \mathrm{s}(n=6000$ tours $/ \mathrm{min})$.

La figure 13 représente les caractéristiques mécaniques de la machine, couplée en fonction de la vitesse, en considérant que l'ensemble des pertes et des inerties internes de la machine ont été ajoutées à la charge, obtenues dans les deux cas suivants :

- Courbe $A: C_{\mathrm{em}}(n) \rightarrow \theta_{\mathrm{m}}=0$ pour $t=0$.

- Courbe $B: C_{\mathrm{em}}(n) \rightarrow \dot{\theta}_{\mathrm{m}}=\dot{\theta}_{\mathrm{m} \max }$ de la courbe A pour $t=0$.

En faisant varier l'inertie, on peut tracer, pour chaque type de fonctionnement (A ou B), un réseau de courbes comme celui de la figure 14 .

Les caractéristiques d'allure semblable sont obte-

ajoutant une charge visqueuse : $D_{\text {ch }}$ par exemple).

La généralisation des résultats à un fonctionnement dynamique établi, avec une charge dissipative extérieure du type frottement visqueux ou sec, montre que l'espace couple-vitesse est toujours borné par deux courbes particulières (Fig. 15) :

- caractéristique limite arrêt-démarrage ou caractéristique de démarrage tout court (pull-in curve) ;

- caractéristique limite en survitesse ou caractéristique d'entraînement (pull-out curve).

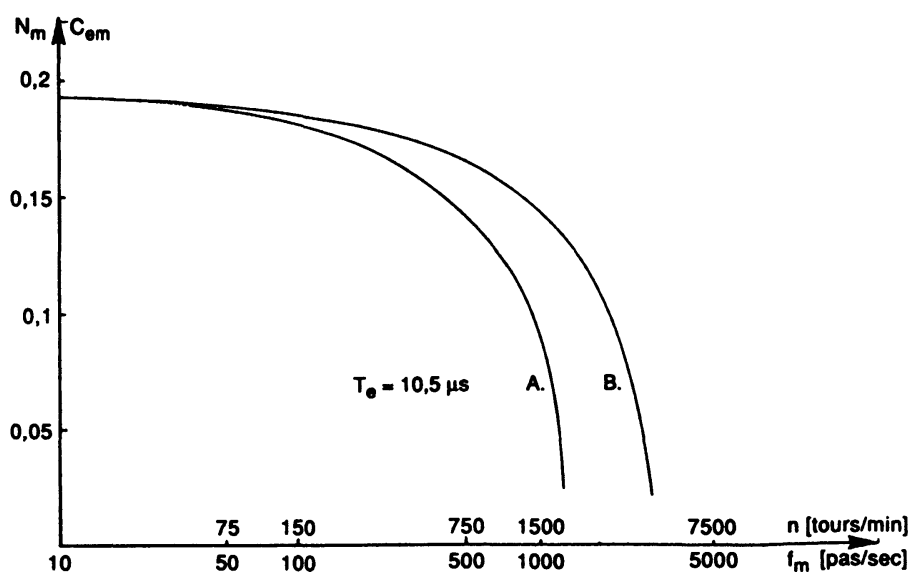

Fig. 13. - Variation du couple avec la fréquence.

[Torque versus frequency.]

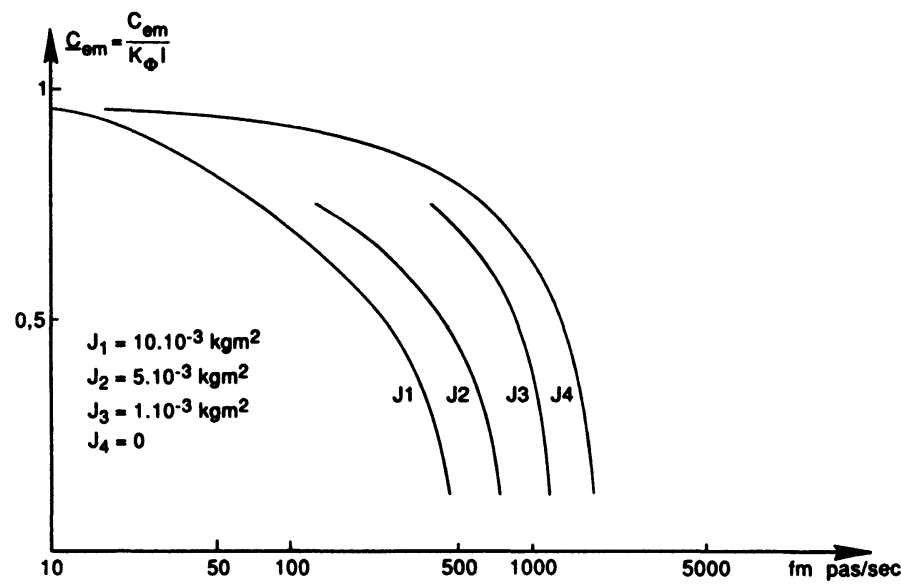

Fig. 14. - Influence de l'inertie totale.

[Influence of the entire inertia.]<smiles>CC1C(C)C1(C)C</smiles>

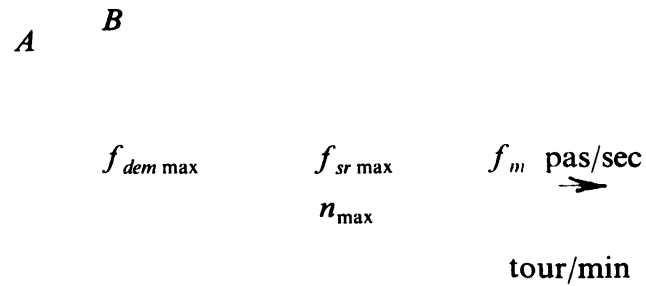

Fig. 15. - Espace du fonctionnement idéalisé.

[Idealized working area.] 
La caractéristique de d'marrage (courbe A) représente le passage du régime statique $\theta_{\mathrm{m}}=\boldsymbol{\eta}$; $\theta_{\mathrm{m}}=0$ ) vers le mouvement à vitesse constante, sans perdre un seul pas. L'intersection de la courbe A avec la droite du couple définit le couple maximal de démarrage, $C_{\text {dem max }}$ (que l'on peut obtenir par ailleurs à partir de l'intersection des deux sinusoïdes consécutives de la caractéristique angulaire du cóuple statique); de même, on détermine la fréquence maximale de démarrage, $f_{\text {dem max }}$.

Lorsque la machine démarre selon la courbe A, on peut, à charge constante, obtenir, par un changement très progressif des fréquences, une vitesse supérieure à celle prédite par la caractéristique de démarrage. La caractéristique d'entraînement (courbe B) ainsi obtenue correspond à un fonctionnement en survitesse sans perte de pas, mais également sans possibilité de démarrage, de freinage, ni de changement de sens de rotation. La caractéristique d'entraînement définit la fréquence maximale des commutations, $f_{\text {sr max }}$, ou encore la survitesse, $n_{\text {max }}$.

L'allure de toutes les courbes indique une diminution du couple électromagnétique en fonction de la fréquence. Cette diminution s'explique par:

- la diminution du courant, due à l'augmentation de l'impédance de l'enroulement en fonction de la fréquence de commutation (donc de la vitesse); tion ;

- la diminution du courant moyen par commuta-

- l'action du couple d'amortissement provenant des f.e.m. de rotation (le terme $D_{\mathrm{r}} / J_{\mathrm{r}} \omega_{\phi} \cdot \mathrm{d} \theta_{\mathrm{e}} / \underline{\mathrm{d} t}$ dans l'expression (43)).

Il est intéressant de mettre en évidence l'influence de la conception de la machine sur ses caractéristiques mécaniques.

La figure 16a montre l'évolution de la caractéristique d'entraînement en fonction de la constante de temps électrique $T_{\mathrm{e}}=L_{0} / R$, où l'on s'aperçoit que la diminution de l'inductance propre et surtout l'augmentation de la résistance du circuit statorique conduisent à l'élargissement de la fréquence de fonctionnement de la machine. Le même effet est obtenu en augmentant le rapport entre le flux propre de l'enroulement et le flux couplé provenant de l'aimant, soit (Fig. 16b) :

$$
k_{\mathrm{cc}}=\frac{K_{\phi}}{p L_{0} I} .
$$

L'augmentation de $k_{\mathrm{cc}}$ et donc de $K_{\phi}$ conduit à l'augmentation de la f.e.m. de rotation et partant des courants induits dans le circuit statorique, comme dans les moteurs synchrones classiques; ce phénomène est à l'origine de l'augmentation des pertes Joule provoquant l'amortissement du mouvement.

Quelle que soit la vitesse de commutation (et donc de rotation), la diminution de $T_{\mathrm{e}}$ contribue à l'augmentation du couple; cette diminution peut être obtenue par des moyens extérieurs, en ajoutant une résistance en série avec le stator ou/et (plus rarement) un condensateur en parallèle, sans parler des alimentations " en courant ". Il n'est pas rare de brancher une résistance «forçante" (forcing resistance), égale à dix foix la résistance des enroulements.

L'ensemble des considérations précédentes illustre bien les calculs numériques des figures $8 \mathrm{f}$ et $8 \mathrm{~g}$.

L'emploi des paramètres $K_{\phi} I, k_{\mathrm{cc}}$ et $T_{\mathrm{e}}$, qui sont soit donnés par le constructeur, soit mesurables, permet de choisir numériquement une série de machines pour le nombre de pas par tour désiré.

Néanmoins, il ne faut pas perdre de vue les limites des raisonnements basés sur les hypothèses très restrictives, ne prenant en compte ni la puissance massique de la machine (qui diminue forcément avec
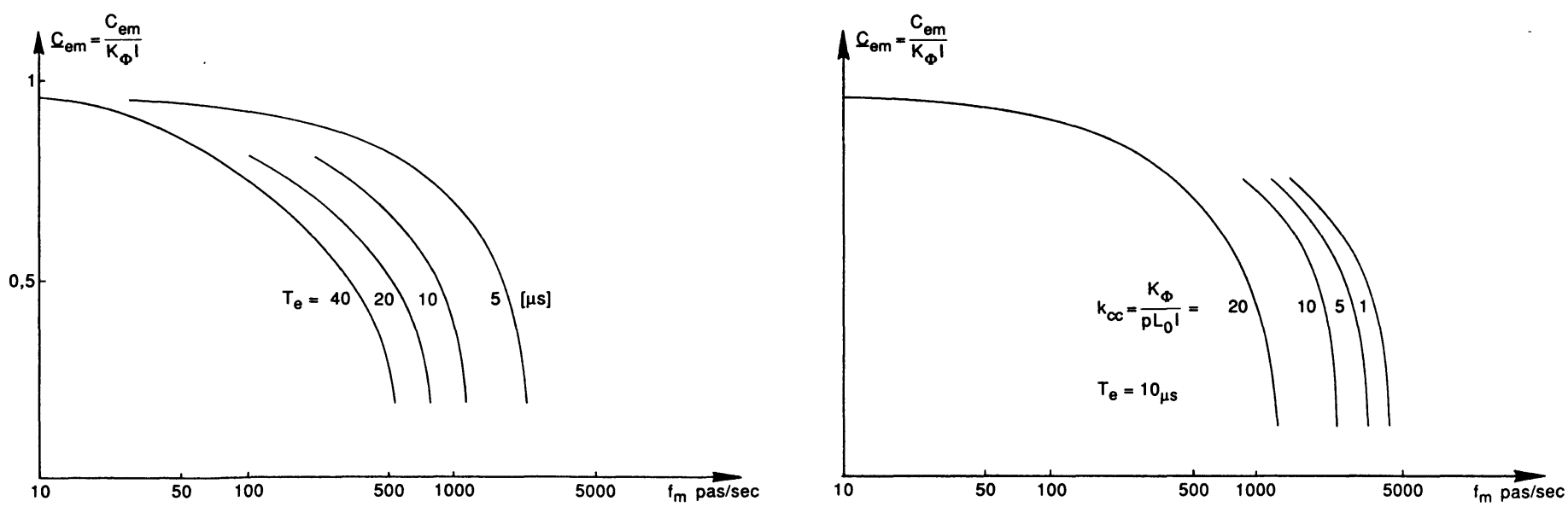

Fig. 16. - (a) Influence de la constante de temps électrique $T_{\mathrm{e}}$. (b) Influence du coefficient de court-circuit $k_{\mathrm{cc}}$.

[(a) Influence of electrical constant $T_{\mathrm{e}}$. (b) Influence of short-circuit constant $k_{\mathrm{cc}}$.] 
le flux d'aimant, donc avec $k_{\mathrm{cc}}$ ), ni les pertes (qui augmentent avec $R$, donc avec la diminution de $T_{\mathrm{e}}$ ), ni surtout les problèmes de stabilité dépendant en grande partie de l'amortissement électromagnétique, donc de $K_{\phi}$.

\section{Conclusion.}

Nous proposons un modèle particulier de la machine pas à pas, muni d'un rotor magnétique bobiné. En supposant, par la suite, que les propriétés de tous les matériaux du dispositif soient indépendantes des valeurs des flux ou/et des courants qu'ils véhiculent, nous établissons les équations du mouvement : électrique et mécanique de la machine dans le repère immobile $\alpha, \beta$ des plots statoriques. La méthodologie utilisée correspond aux investigations classiques des machines synchrones; la modification de la perméabilité et de la conductivité du rotor permet de tenir compte à la fois des machines à rotor actif (aimant) et à rotor passif (fer denté). Pour s'affranchir des fonctions trigonométriques du repère immobile, nous passons au repère mobile $a, b$ (vecteur tournant lié au rotor), permettant d'obtenir les équations du mouvement aisément programmables. Enfin, la transformation des équations du repère mobile, par l'introduction de grandeurs sans dimen- sions, montre clairement une structure physique unique de toutes les machines pas à pas et donc le même ordre de réponses qu'elles doivent présenter aux sollicitations électriques ou/et mécaniques. Les équations adimensionnelles, convenablement analysées, peuvent fournir beaucoup de renseignements sur les modes d'alimentation, sur le positionnement et l'accélération des machines pas à pas. Comme les équations du mouvement sont non linéaires, leur solution ne peut être que numérique. Ainsi, une partie du mémoire est consacrée à l'analyse des fonctions transitoires, tracées par l'ordinateur. On peut ainsi mettre en évidence un certain nombre de propriétés de la machine, qui, dans la plupart des cas, recoupent les études analytiques plus restrictives, ou encore les expérimentations largement diffusées dans la littérature technique.

Comme les méthodes performantes de relaxation numérique sont actuellement très accessibles aux micro-ordinateurs, notre travail doit permettre, d'une part, une étude plus approfondie des cas particuliers de fonctionnement des machines pas à pas et, d'autre part, la comparaison des différents types de ces machines. Enfin, ce qui n'est pas négligeable, les formulations proposées peuvent servir d'outil didactique pour la formation de spécialistes dans le domaine des actionneurs électriques.

\section{Bibliographie}

[1] Steps motors and control systems, Travail collectif sous la rédaction de B. C. Kuo (S.R.L. Publising Company) 1979.

[2] Eid G., Mouillet A., Transistorized DC brushless micromotor with rare earth permanent magnets, Proc. ICEM (1984).

[3] KANT M., Actionneurs électriques pas à pas (Editions Hermès) 1989.

[4] Adxins B., Harley R. G., The general theory of alternating current machine (Chapman Hall London) 1978.

[5] Pinchon O., Gruniaux P., Brienne J. P., Povy L., Commande d'un moteur pas à pas en boucle ouverte. Influence du modèle d'étude, $5^{\mathrm{e}}$ colloque sur les moteurs pas à pas, Nancy (1988).

[6] Creuzet T., Abignoli M., Calcul du couple dynamique d'un moteur pas à pas, $5^{\mathrm{e}}$ colloque sur les moteurs pas à pas, Nancy (1988).

[7] Yonnet J. P., Guiresse L. R., Chillet C., Etude des pertes dans un moteur pas à pas à aimant permanent, $5^{\mathrm{e}}$ colloque sur les moteurs pas à pas, Nancy (1988).

[8] Sargos F. M., Machines à réluctance à plots nonexcitées. Optimisation des structures. Conséquences sur le fonctionnement et l'alimentation, $5^{\mathrm{e}}$ colloque sur les moteurs pas à pas, Nancy (1988). 